\title{
A Near-Chromosome Level Genome Assembly of the European Hoverfly, Sphaerophoria Rueppellii (Diptera: Syrphidae), and a Comparative Analysis of Insecticide Resistance-Related Gene Families in Hemipteran Crop Pests and Pollinators
}

Emma Bailey ( $\square$ emma.bailey@rothamsted.ac.uk)

Rothamsted Research, Biointeractions and Crop Protection

Linda Field

Rothamsted Research, Biointeractions and Crop Protection

Christopher Rawlings

Rothamsted Research, Computational and Analytical Sciences

Rob King

Rothamsted Research, Computational and Analytical Sciences

Fady Mohareb

Cranfield University, Environment and Agrifood

Keywan Hassani-Pak

Rothamsted Research, Computational and Analytical Sciences

\section{David Hughes}

Rothamsted Research, Computational and Analytical Sciences

\section{Martin Williamson}

Rothamsted Research, Biointeractions and Crop Protection

\section{Eric Ganko}

Syngenta Crop Protection, Seeds Research, LLC, Triangle Research Park

\section{Benjamin Buer}

Bayer AG, Crop Science Division, R\&D

\section{Ralf Nauen}

Bayer AG, Crop Science Division, R\&D

\section{Research Article}

Keywords: Sphaerophoria rueppellii, hoverfly, PacBio, Illumina, Hi-C, Whole genome sequencing, beneficial predator, insecticide resistance, comparative genomics, diptera, crop pests

Posted Date: November 17th, 2021

DOI: https://doi.org/10.21203/rs.3.rs-1050342/v1

License: (c) (i) This work is licensed under a Creative Commons Attribution 4.0 International License. Read Full License 


\section{Abstract}

Background: Sphaerophoria rueppellii, a European species of hoverfly, is a highly effective beneficial predator of crop pests including aphids, thrips and coleopteran/lepidopteran larvae in integrated pest management (IPM) programmes. It is also a key pollinator of a wide variety of important agricultural crops. No genomic information is currently available for S. rueppellii. Without genomic information for such beneficial predator species, we are unable to perform comparative analyses of insecticide target-sites and genes encoding metabolic enzymes potentially responsible for insecticide resistance, between crop pests and their predators. These metabolic mechanisms include several gene families cytochrome P450 monooxygenases (P450s), ATP binding cassette transporters (ABCs), glutathione-S-transferases (GSTs), UDP-glycosyltransferases (UGTs) and carboxyl/choline esterases (CCEs).

Methods and findings: In this study, a high-quality near-chromosome level de novo genome assembly (as well as a mitochondrial genome assembly) for $S$. rueppellii has been generated using a hybrid approach with PacBio long-read and Illumina short-read data, followed by super scaffolding using Hi-C data. The final assembly achieved a scaffold N50 of $87 \mathrm{Mb}$, a total genome size of $537.6 \mathrm{Mb}$ and a level of completeness of $96 \%$ using a set of 1,658 core insect genes present as full-length genes. The assembly was annotated with 14,249 protein-coding genes.

Comparative analysis revealed gene expansions of CYP6Zx P450s, epsilon-class GSTs, dietary CCEs and multiple UGT families (UGT37/302/308/430/431). Conversely, ABCs, delta-class GSTs and non-CYP6Zx P450s showed limited expansion. Differences were seen in the distributions of resistance-associated gene families at the subfamily levels between $S$. rueppellii and some crop pests.

Conclusion and significance: This assembly is the first published genome for a predatory member of the Syrphidae family and will serve as a useful resource for further research into selectivity and potential tolerance of insecticides by beneficial predators. Furthermore, the expansion of some gene families often linked to insecticide resistance and selectivity may be an indicator of the capacity of this predator to detoxify IPM selective insecticides. These findings could be exploited by targeted insecticide screens and functional studies to increase effectiveness of IPM strategies, which aim to increase crop yields by sustainably and effectively controlling pests without impacting beneficial predator populations.

\section{Introduction}

Loss of crops to insect pests can account for more than $10 \%$ of potential yield, as a result of both direct feeding damage and the transfer of plant viruses via insect feeding [1]. Methods of controlling insect pests are therefore critical to ensure that crop yields are maximised to sustain the growing world population. Insecticides play a key role in pest control strategies. Many modern insecticides are known to be selective for pests without harming beneficials. However, some insecticides such as pyrethroids tend to be non-specific, and as a result are often toxic to both their target pest species and beneficial predators. Applications of such non-specific insecticides can reduce predator populations so that they are unable to act as an effective natural control. This can lead to pest populations surging, with instances of higher populations than pre-pesticide application [2-4].

Beneficial predators, such as those in the Syrphid family, are effective in the biological control of crop pests. Syrphid adults typically feed on nectar and pollen, however, the larvae of roughly one-third of syrphid species feed on crop pests such as aphids, thrips and coleopteran and lepidopteran larvae [5-11]. Predatory Syrphidae are able to feed on up to $\sim 500$ aphids during their larval stage, which is a higher daily feeding rate than other aphid predators [12]. For example, $S$. rueppellii were able to reduce aphid (Myzus persicae) populations by $84 \%$ in a field experiment [13]. Specialised adaptations present within adult female Syrphidae allow them to detect aphid pheromones and increase their efficacy as biological control agents - for example, adult females often lay their eggs in close proximity to aphid colonies to ensure a plentiful food supply for emerging larvae [14]. Syrphid adults also avoid laying their eggs close to parasitised aphids [15] 
which reduces intraguild predation between parasitoids and hoverflies, and thus allows for them to be safely combined in IPM strategies. Such strategies can result in more effective pest control compared to using only one beneficial predator species, especially when attempting to control more than one species of pest [16]. Overall, it is unsurprising that Syrphidae are considered to be amongst the most important aphid predators and a key tool for biological control $[17,18]$.

Alongside pest control, adult hoverflies play a key role in pollination [19] and are considered the second most important pollinator after the Apidae bee families [20]. Unlike bees, hoverflies are highly migratory and therefore capable of transporting pollen over long distances - which has benefits for both the plants and other non-migratory pollinators [21]. Pollination experiments have showed that hoverflies increase seed number in food crops such as strawberry, oilseed rape and sweet pepper (which also showed increased fruit abundance) [13,22, 23].

This dual role as effective pollinators and biological control agents [11] makes hoverflies hugely attractive for commercial use and also highlights the need to develop IPM strategies which conserve their populations. The aim of this work was to produce a high-quality genome assembly for $S$. rueppellii, to serve as a resource for research into this species as well as the wider Syrphidae family, which consists of $\sim 6000$ species worldwide $[19,24]$ and is therefore a potentially valuable source of biological control agents.

The number of beneficial predator genomes has been trailing behind crop pest genomes in recent years, although numbers are now on the rise, especially with the progress being made by the Darwin tree of Life (DToL) sequencing project. High quality genomes have already been released by DToL for some beneficial predators such as green lacewing (Chrysoperla carnea) and the seven spotted ladybird (Coccinella septempunctata) [25]. Other publicly available beneficial predator genomes include: a phytoseiid mite, parasitoid wasps, a minute pirate bug and lady beetles [26-29]. To date the only available genome for the Syrphid family is the non-predatory European hoverfly (Eristalis pertinax) released by DToL (but not yet annotated at the time of writing), so this $S$. rueppellii genome is the first available for a predatory member of the Syrphid family.

The EU Directive on Sustainable Use of Pesticides 2009/128/EC [30] means that IPM strategies, including the use of beneficial predators [31-35], are growing in necessity. These strategies can be supported by comparative analyses of the genomes of predators and pests, focusing on potential differences in insecticide tolerance mechanisms based on both target-site selectivity and metabolism.

There are two main types of insecticide resistance mechanisms: mutations in insecticide target genes that prevent the insecticide binding to the target [36] and duplication or increased expression of genes encoding enzymes which can metabolise insecticides. Gene families associated with metabolic resistance include cytochrome P450 monooxygenases (P450s), ATP binding cassette transporters (ABCs), glutathione S-transferases (GSTs), UDP-glycosyltransferases (UGTs) and carboxyl/choline esterases (CCEs) [37-42]. Comparisons of these mechanisms in beneficial predators and crop pests could help identify insecticides which will target crop pests but have limited impact on beneficial predator populations. This information could prove key to developing successful IPM strategies which exploit differences in insecticide selectivity between the predator and crop pests. Improving the availability of beneficial predator genomes could also aid the selection of beneficial predators with genes/mutations for insecticide resistance before being released in the field for biological control [43].

The results presented here provide a comprehensive foundation for further study of insecticide tolerance and selectivity mechanisms in beneficial predators and how they compare to crop pests.

\section{Materials And Methods}

\section{Sample preparation and sequencing}


S. rueppellii larvae were obtained from 'biopestgroup.com'. $\mathrm{CO}_{2}$ was used for anaesthesia to allow the insects to be sorted from the substrate. The larvae were then flash frozen with liquid $\mathrm{N}_{2}$ and stored at $-80^{\circ} \mathrm{C}$. The whole process was completed within 48 hours of arrival.

For transcriptome sequencing, RNA extractions were carried out in-house at Rothamsted Research using the Bioline Isolate

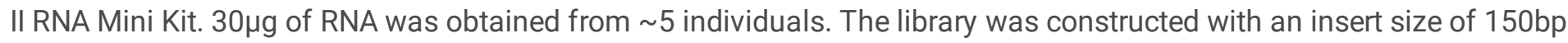
and PolyA selection for rRNA removal. Sequencing was performed by Genewiz (New Jersey, US) using Illumina HiSeq 4000 with a 2x150bp paired-end configuration.

For short-read genomic sequencing, DNA extractions were performed in-house at Rothamsted Research using the commercial DNAzol reagent. Short reads were sequenced using $1.1 \mu \mathrm{g}$ of DNA obtained from $\sim 5$ individuals and a library with an insert size of 200bp. Sequencing was performed by Genewiz (New Jersey, US) using Illumina HiSeq 4000 with a 2x150bp paired-end configuration. K-mer counting of the raw Illumina DNA data was performed using Jellyfish 2.2.6 [44]. Canonical (-C) 21-mers (-m 21) were counted and a histogram of k-mer frequencies outputted. GenomeScope 2.0 [45] was used to process this histogram with ploidy set to 2 and maximum k-mer coverage cut-off set to 10,000.

For long-read genomic sequencing, whole insects were sent directly to Georgia Genomics (University of Georgia, US) who performed the DNA extractions using 15 individuals. To obtain long-read PacBio data, a 15-30Kb SMRTbell library was produced with an insert size of 24,000bp and a 15 hour sequencing run was carried out using PacBio Sequel II.

For Hi-C sequencing, whole insects were sent directly to Arima Genomics (San Diego, US) who carried out the DNA extractions using 10 individuals. Arima-QC and library preparation were also performed in-house. Sequencing was performed using Illumina HiSeq X with a 2 x 150bp paired-end configuration.

\section{Genome quality assessment}

Basic metrics from the genome assembly were calculated using a script developed for the 'Assemblathon' [46]. These metrics include scaffold/contig N50, longest and shortest scaffold length, number of scaffolds exceeding a range of lengths and number of gaps/N's in the assembly.

The completeness of the genome assembly and annotation for S. rueppellii was assessed using the Benchmarking Universal Single-Copy Orthologs (BUSCO) [47] of the insect gene set (insecta odb9). 'Genome' mode was used to assess the assembly, and 'protein' mode to assess the annotation. 'Fly' was used as the training species for Augustus gene prediction. BUSCO assessments were then run with default parameters.

\section{De novo genome assembly}

FastQC v.0.11.8. [48] was used to perform quality checks on the raw Illumina HiSeq DNA and RNA sequence data. Adapters were trimmed, low-quality bases (below a score of 3) were removed from the start and end of reads and any reads with a length less than 36 bases were also removed. Trimmomatic v.0.38. [49] was used for these trimming steps.

The raw PacBio reads were subsetted using a 'SelectLongestReads' script from:

https://github.com/yechengxi/AssemblyUtility to reduce coverage from $277 x$ to $150 x$ coverage prior to assembly. The subsetted PacBio long reads were then assembled into contigs with the Flye v2.5. de novo assembler [50,51] with the following parameters: '-genome-size 300m -i 3 -meta'. This subsetting was used to reduce duplication in the assembly outputted by Flye whilst maintaining the completeness of the genome.

The subsetted PacBio long reads and Illumina DNA short reads were also assembled into contigs using Platanus Allee v2.2.2 [52] with default parameters. This is a hybrid assembler designed for heterozygous data. 
QuickMerge v0.3 [53] was used to merge the Flye and Platanus-Allee assemblies, with Flye as the reference assembly. BUSCO outputs were compared between the merged assembly and the standalone assemblies to identify core insect genes which had been lost during the merging process. Full-length contigs containing these missing genes were extracted from the standalone assemblies and added to the merged assembly, based on the assumption that these contigs would also contain other missing genes (i.e. those not included in BUSCO's list of 1,658 core insect genes).

Purge Haplotigs v1.0.0 [54] was next used to perform redundant contig removal from the merged assembly. Parameters '-I 5 -m 30 -h 190' were chosen from the coverage histogram outputted in the first step of the pipeline. The percent cutoff for identifying a contig as a haplotig was set to '-a 40', (the default value is 70 , however a lower cutoff was chosen due to a very high level of duplication). This tool takes read depth coverage into consideration to reduce over-purging of repetitive regions and paralogous contigs, whilst still coping well with highly heterozygous assemblies.

The Hi-C data was processed using Juicer v1.5 [55] and used as input to the 3D-DNA de novo genome assembly pipeline (version 180922) [56] alongside the draft assembly to produce a candidate chromosome-length genome assembly. Contact matrices were generated by aligning the $\mathrm{Hi}-\mathrm{C}$ dataset to the genome assembly after Hi-C scaffolding, and were then visualised using JuiceBox Assembly Tools v1.11.08 [57]. The parameters used were as follows: '--mode haploid -build-gapped-map -sort-output'. Additional finishing on the scaffolds was performed in JuiceBox to correct mis-joins.

Multiple rounds of Pilon [58] error polishing were performed, using the Illumina short read data, until no further improvement in BUSCO score was seen. A final round of Purge Haplotigs was then performed to reduce duplication further. Parameters '-I 10 -m 50 -h 150' were chosen from the coverage histogram outputted in the first step of the pipeline. The percent cutoff for identifying a contig as a haplotig was set to '-a 80 '.

\section{Mitochondrial genome assembly}

The mitochondrial genome was found and extracted by running a BLAST search of the $S$. rueppellii genome against the Syrphus ribesii mitochondrial genome, which is publicly available at NCBI, GenBank accession number: MW091497.1.

\section{Annotation}

Gene prediction was performed using the MAKER v2.31.8 pipeline [59] through the incorporation of both transcriptome evidence and $a b$ initio gene prediction as well as a custom repeat library (see below). MAKER was run using Augustus v3.3.1 [60], GeneMark-ES v4.32 [61] and FGeneSH v8.0.0 [62] as well as EVidenceModeler v1.1.1 [63] with default masking options.

A de novo species specific repeat library was constructed using RepeatModeller v1.0.7 [64] to identify repeat models. These models were searched against the GenBank non-redundant ( $n r)$ protein database for Arthropoda (e value $<10^{-3}$ ) using Blastx to remove any potential protein-coding genes. This was combined with transposon data to create a custom library. Transposons were identified from the transcriptome assembly by running HMMER: hmmscan [65] against the Pfam database [66] and filtering the resultant Pfam descriptions for those containing "transposon". A search for transposons was also performed on transcripts produced from MAKER and these transposons were then added to the custom repeat library which was used for a second round of MAKER. RepeatMasker v4.0.7 [67] was used to mask repeats in the genome assembly using these repeat libraries, as well as to estimate the abundances of all predicted repeats.

RNA-seq reads were mapped to the genome with HISAT2 v2.0.5 [68] for assembly with StringTie v1.0.1 [69]. A de novo assembly was also done using Trinity v2.5.1 [70]. The best transcripts were selected from the Trinity and StringTie assemblies using Evigene v19.jan01 [71].

Evidence from assembled transcripts was transferred to the genome assembly via MAKER. The output from this was then used to produce a high confidence level gene model training set. Overlapping and redundant gene models were removed. 
Augustus and GeneMark were trained using this training set prior to being used for $a b$ initio gene predictions. FGeneSH was run based on the Drosophila melanogaster genome.

The best transcripts (classified by reasonable transcript size and homology to other species) from both the ab initio gene prediction annotation and the transcriptome-based annotation were selected using Evigene and combined to create the final annotation.

S. rueppellii protein sequences were aligned using Blastp against the non-redundant (nr) NCBI protein database for Arthropoda. InterProscan searches were run against several databases (CDD, HAMAP, HMMPAnther, HMMPfam, HMMPIR, FPrintScan, BlastProDom, ProfileScan, HMMTigr) for functional annotation. BLAST2GO [72] was used to assign gene ontology (GO annotations). Infernal v1.1.2 [73] was used to predict and annotate non-coding RNAs.

The mitochondrial genome was annotated using MITOS2 [74] with reference database 'RefSeq 89 Metazoa' and genetic code '5 Invertebrate'.

\section{Comparative genomics and phylogenetic analysis}

To produce the species tree, orthogroup gene trees were produced using OrthoFinder [75] and the tree was inferred from these using the STAG method [76].

In order to identify genes potentially involved in insecticide resistance, the PFAM domains assigned to gene models during annotation (as described in the 'Genome Annotation' methods section) were used as follows: CCEs (PF00135/IPR002018), GSTs (IPR004045/PF02798), (IPR004046/PF00043), P450s (IPR001128/PF00067), ABCs (IPR003439/PF00005) and UGTs (IPR002213/PF00201). Proteins from UniProtKB for the classes of interest, from hemipteran species, were used for BLAST queries against $S$. rueppellii to identify any missed genes and to assist with subfamily assignment within these classes. Subfamily assignment for $S$. rueppellii gene families was finalised using phylogenetic trees which were produced using MAFFT alignments [77, 78] and RaxML v8.2.11 [79]. The GAMMA LG protein model [80] was used and a bootstrap consensus tree was inferred from 100 replicates.

Manual checks and curation were performed for genes potentially involved in insecticide resistance. Increased copy numbers of genes linked to insecticide resistance often led to adjacent tandem duplications being incorrectly annotated by MAKER as one gene model; therefore curation was important to prevent incorrect gene numbers being reported in later analyses. The exon/intron boundaries and start/stop codons of the genes were confirmed through visualization in IGV [81] of RNAseq data mapped to the genome using HISAT2 v2.0.5 [68] and the gene models were edited in Geneious where necessary.

The P450s were classified and named by Dr David Nelson [82]. The UGTs were classified and named by Dr Michael Court [83]. Nomenclature of P450s and UGTs is based on the evolutionary relationships of the sequences. P450 and UGT sequences were BLAST searched against named insect sequences and were assigned to known families if they were $>40 \%$ (for P450 families) or >45\% (for UGT families) identical. Other sequences were assigned to new families based on their clustering on trees and their percent identity to each other.

\section{Results And Discussion Sequencing}

30 individuals of S. rueppellii were required to produce sufficient DNA and RNA for sequencing. Since they were obtained commercially, the level of inbreeding of the culture was not known. However, all individuals were obtained from a single colony within the rearing facility. A high heterozygosity level was therefore a possibility and this was kept in mind during the assembly process. 


\section{Raw data}

The DNA sequencing generated 6,748,327 PacBio reads with a total length of $83.2 \mathrm{Gbp}(277 \mathrm{x})$ and a polymerase read length $\mathrm{N} 50$ of $63,285 \mathrm{bp}$.

A total of $125.3 \mathrm{~Gb}$ was produced from the Illumina HiSeq platform for whole genome sequencing, as well as $36.9 \mathrm{~Gb}$ for transcriptome sequencing. Quality trimming of Illumina reads using Trimmomatic to remove adapters and any reads $<36 \mathrm{bp}$ resulted in a $2.9 \%$ loss of reads for whole genome sequencing and a $5.18 \%$ loss of reads for transcriptome sequencing (Table 1).

Table 1

Number of paired-end Illumina HiSeq DNA/RNA sequences present before and after trimming using Trimmomatic. Adapters and reads $<36 \mathrm{bp}$ were removed.

\begin{tabular}{|lll|}
\hline & Illumina DNA Reads & Illumina RNA Reads \\
\hline Total sequences before trimming & $417,662,063$ & $123,298,454$ \\
\hline Total sequences after trimming & $405,634,072$ & $116,917,664$ \\
\hline Sequences lost & $12,027,991$ & $6,380,790$ \\
\hline
\end{tabular}

A total of $21.6 \mathrm{~Gb}$ of sequencing data was produced from Arima-HiC. Analysis of proximal ligation gave a library QC metric of $30 \%$ (a high-quality Arima-HiC library is $>15 \%$ ).

\section{Genome metrics evaluation based on raw reads}

The raw read k-mer analysis with GenomeScope 2.0 (see figure 1) estimated a haploid genome size of $\sim 400 \mathrm{Mb}$ (Table 2), which is an underestimate of the final assembly size of $537 \mathrm{Mb}$. However, such discrepancies are often seen when using kmer frequency to estimate genome size in genomes with high repeat content and high heterozygosity [84]. Genome repeat length was $170 \mathrm{Mb}, 42 \%$ of the total estimated genome size.

Table 2

Genome characteristics obtained from GenomeScope v2.0. Using a k-mer length of 21, ploidy set at 2 and a maximum k-mer coverage of 10,000

\begin{tabular}{|lll|}
\hline Genome characteristics & Minimum & Maximum \\
\hline Heterozygosity, \% & 3.235 & 3.356 \\
\hline Genome Haploid Length (Mb) & 397.7 & 403.1 \\
\hline Genome Repeat Length (Mb) & 169.1 & 171.4 \\
\hline Genome Unique Length (Mb) & 228.6 & 231.7 \\
\hline Read Error Rate, \% & 0.153 & 0.153 \\
\hline
\end{tabular}

The heterozygosity rate ranged from $3.24-3.36 \%$. This indicates a fairly high level of heterozygosity, which was taken into consideration in the assembly strategy.

\section{Assembly}


Flye and Platanus-Allee were used to produce 2 separate assemblies. Flye had the best assembly statistics in terms of scaffold N50 (100,207bp with 18 scaffolds >1 million bp) and BUSCO completeness score (99.2\%), however, duplication was very high (48.3\%) for this assembly, even after subsetting the longest reads to get 150x coverage (duplication was $63.8 \%$ prior to subsetting). The total number of scaffolds was 50,164 . Platanus-Allee had a lower scaffold N50 (42,845bp with 0 scaffolds $>1$ million bp) and a slightly lower BUSCO completeness score (97.6\%), however, duplication was much lower (3.6\%). The total number of scaffolds was 67,142 .

The Flye and Platanus-Allee assemblies were merged using QuickMerge, and some manual curation was performed to bring back falsely removed contigs. This resulted in an assembly with a completeness score of $96.5 \%$, duplication of $15.5 \%$, a scaffold $\mathrm{N} 50$ of $67,653 \mathrm{bp}$ and a total of 59,284 scaffolds, 16 of which were $>1$ million bp.

A subsequent round of Purge Haplotigs brought the duplication score down to $4.6 \%$ whilst still maintaining a completeness of $95.6 \%$. Scaffold N50 increased to $126,450 \mathrm{bp}$ and the total number of scaffolds was reduced to 15,009 .

This draft assembly was next used for scaffolding with Hi-C data using the 3D-DNA de novo genome assembly pipeline. This increased the scaffold N50 to $87,361,475 \mathrm{bp}$, with 5 scaffolds > 10 million bp. The total number of scaffolds was reduced to 11,549, with 6 chromosomal-level scaffolds, numbered by sequence length (figure 2). The BUSCO completeness score was reduced to $94.6 \%$, however, a round of Pilon error polishing brought this back up to $96.4 \%$ (subsequent rounds of Pilon worsened the BUSCO score). A final run with Purge Haplotigs reduced duplication from 4$3 \%$. Statistics of the final assembly are shown in Table 3.

Table 3

Final assembly statistics for the $S$. rueppellii genome

\begin{tabular}{|ll|}
\hline Number of scaffolds & $\mathbf{8 , 4 7 6}$ \\
\hline Total size of scaffolds & $537,631,316 \mathrm{bp}$ \\
\hline Longest scaffold & $125,413,692 \mathrm{bp}$ \\
\hline Shortest scaffold & $957 \mathrm{bp}$ \\
\hline Number of scaffolds $>1 \mathrm{~K} \mathrm{nt}$ & $8,412(99.2 \%)$ \\
\hline Number of scaffolds $>10 \mathrm{~K} \mathrm{nt}$ & $2,095(24.7 \%)$ \\
\hline Number of scaffolds $>100 \mathrm{~K}$ nt & $70(0.8 \%)$ \\
\hline Number of scaffolds $>1 \mathrm{M} \mathrm{nt}$ & $9(0.1 \%)$ \\
\hline Number of scaffolds $>10 \mathrm{M} \mathrm{nt}$ & $5(0.1 \%)$ \\
\hline N50 scaffold length & $87,097,991 \mathrm{bp}$ \\
\hline Number of N's & $56,988,920$ \\
\hline BusCO & C:96.0\%[S:93.0\%,D:3.0\%], F:1.2\%,M:2.8\% \\
\hline
\end{tabular}

The final assembly size of $537.6 \mathrm{Mb}$ was slightly larger than the assembled genome size for E. pertinax (482Mb) [85], but closely matches the genome size for Episyrphus balteatus $(530 \mathrm{Mb})$ from the Syrphidae family, which was calculated using flow cytometry and can therefore be considered a more accurate estimate [86].

\section{Annotation}


Gene prediction with MAKER identified 14,249 protein-coding genes with the proteins having a mean length of 465 amino acids. Of these, 10,789 (76\%) had a match to NCBI's non-redundant ( $n r)$ database and 12,000 (84\%) contained InterPro motifs, domains or signatures. The longest protein found was a 'nesprin-1 isoform' at 17,083aa. The final proteome had a BUSCO completeness score of $87.3 \%$ (with $4.9 \%$ duplication).

From the Infernal tool inference of RNA alignments, a total of 2,292 non-coding RNA elements were found in the genome (Table 4).

Table 4

Number of ncRNAs predicted in the $S$. rueppellii genome

\begin{tabular}{|ll|}
\hline ncRNA element & Number of elements \\
\hline tRNA & 2,058 \\
rRNA & 37 \\
\hline snRNA & 79 \\
miRNA & 81 \\
\hline srpRNA & 7 \\
\hline snoRNA & 28 \\
\hline IncRNA & 2 \\
\hline
\end{tabular}

\section{Repeat annotation}

Transposable and repetitive elements made up $30 \%$ of the $S$. rueppellii genome (Table 5 ). This is consistent with the reported repeat content of genomes of Diptera species, which ranges widely from $7 \%$ (Drosophila simulans) to $55 \%$ (Aedes aegypti) [87]. $16.15 \%$ of the S. rueppellii genome (77,619,601bp) was masked for annotation - some repeats are annotated but not masked, such as those less than 10bp in length. The majority of these were LINES (9.97\%) and interspersed repeats (14.35\%). Details of transposable and repetitive elements are shown in Table 5. 
Table 5

Summary of transposable and repetitive elements in the $\mathrm{S}$. rueppellii genome. Percentages do not include runs of $\mathrm{X} / \mathrm{Ns}>=20$

\begin{tabular}{|c|c|c|c|}
\hline & Number of Elements & Length Occupied (bp) & Percentage of Sequence \\
\hline Retroelements & 83,508 & $57,419,755$ & 11.94 \\
\hline SINES & 21 & 6045 & 0.00 \\
\hline Penelope & 114 & 59,699 & 0.01 \\
\hline LINES & 75,547 & $47,912,743$ & 9.97 \\
\hline L2/CR1/Rex & 10,835 & $4,939,603$ & 1.03 \\
\hline R1/LOA/Jockey & 659 & 459,946 & 0.10 \\
\hline $\mathrm{R} 2 / \mathrm{R} 4 / \mathrm{NeSL}$ & 37 & 26,828 & 0.01 \\
\hline RTE/Bov-B & 56,568 & $39,492,974$ & 8.22 \\
\hline LTR elements & 7,490 & $9,500,967$ & 1.98 \\
\hline $\mathrm{BEL} / \mathrm{Pao}$ & 1,433 & $2,121,736$ & 0.44 \\
\hline Ty1/Copia & 2,122 & $2,534,993$ & 0.53 \\
\hline Gypsy/DIRS1 & 3,935 & $4,844,238$ & 1.01 \\
\hline DNA transposons & 16,586 & $8,092,263$ & 1.68 \\
\hline hobo-Activator & 88 & 35,361 & 0.01 \\
\hline Tc1-IS630-Pogo & 16,004 & $7,819,876$ & 1.63 \\
\hline PiggyBac & 27 & 8,274 & 0.00 \\
\hline Other (Mirage, P-element, Transib) & 23 & 13,960 & 0.00 \\
\hline Rolling-circles & 3,616 & 462,376 & 0.10 \\
\hline Unclassified & 6,299 & $3,448,721$ & 0.72 \\
\hline Total interspersed repeats & - & $68,960,739$ & 14.35 \\
\hline Small RNA & 54 & 15,691 & 0.00 \\
\hline Satellites & 11 & 817 & 0.00 \\
\hline Simple repeats & 150,503 & $6,316,344$ & 1.31 \\
\hline Low complexity & 36,195 & $1,890,143$ & 0.39 \\
\hline Total & 296,772 & $146,606,849$ & 30.00 \\
\hline
\end{tabular}

\section{Mitochondria}

A circularized mitochondrial genome of 16,387bp, assembled and annotated using MITOS2, consisted of 13 protein coding genes, 22 tRNA genes, 2 rRNA genes and an A+T rich region with a length of 1,500bp (figure 3 ). This is very similar to the Syrphus ribesii mitochondrial genome which is $16,530 \mathrm{bp}$ in length and also has 13 protein coding genes, 22 tRNA genes, 2 rRNA genes and an A-T rich region [88]. 
OrthoFinder assigned 435,592 genes ( $93.6 \%$ of total) to 28,834 orthogroups. There were 1,805 orthogroups with all species present and one of these consisted entirely of single-copy genes. Phylogenetic analysis correctly clustered $S$. rueppellii within the dipteran clade, between the Phoridae and Drosophilidae families [89] (figure 4).

\section{Comparative genomics}

UDP-glycosyltransferases

UDP-glycosyltransferases (UGTs) are phase II detoxification enzymes which are involved in insecticide metabolism. The mechanisms of UGT-mediated resistance are for example based on the conjugation of P450-functionalized substrates, however, their upregulation has been shown in resistant strains of $P$. xylostella [37], and they have been linked to diamide resistance in Chilo suppressalis [90] and neonicotinoid resistance in Diaphorina citri [91] and they also contribute to insecticide detoxification via the elimination of oxidative stress in Apis cerana [92].

Table 6

Numbers of annotated UDP glucosyltransferase genes found in Sphaerophoria rueppellii (this study), Drosophila melanogaster [93], Anopheles sinensis, Aedes aegypti, Anopheles gambiae [94], Apis mellifera, Bombus impatiens, Bombus huntii [95], Tetranychus urticae, Nilaparvata lugens, Acyrthosiphon pisum, Bemisia tabaci [96], Myzus persicae [97], Trialeurodes vaporariorum [98] and Thrips palmi [99].

\begin{tabular}{|lllllllllllllllll|l|}
\hline \multicolumn{10}{|c}{ S. rueppellii + close relatives } & \multicolumn{1}{c|}{ Pollinators } & \multicolumn{1}{c|}{ Crop pests } \\
& Sr & Dm & As & Aa & Ag & Am & Bi & Bh & Tu & Nl & Mp & Ap & Tv & Bt & Tp \\
\hline Total & 46 & 35 & 30 & 32 & 23 & 2 & 8 & 2 & 81 & 20 & 101 & 72 & 55 & 76 & 17 \\
\hline
\end{tabular}

There are 46 UGTs in the S. rueppellii genome (Table 6), which are classified into 14 families as shown in figure 5 (UGT36, UGT37, UGT49, UGT50, UGT301, UGT302, UGT308, UGT314, UGT316, UGT430, UGT431, UGT432, UGT433, UGT435). Of these families, UGT430-435 are species specific to $S$. rueppellii, whilst all other families are present in at least one other Diptera species [94].

The UGT genes are distributed across predicted chromosomes 1-5 (with the exception of 1 gene, which is located on a scaffold additional to the chromosome superscaffolds) and the majority (26) are on potential chromosome 2. 38 of the genes are located within clusters of 2-13 tandem UGT genes which generally consist of genes from the same UGT family. This indicates that a high degree of tandem duplication within the UGT gene family likely occurred in $S$. rueppellii.

39 out of 46 UGT genes belong to 7 of the UGT families (UGT308, UGT36, UGT49, UGT302, UGT430, UGT37 and UGT431), suggesting a significant lineage-specific expansion within these 7 families. There is a greater degree of UGT lineage specific expansion and subsequently a higher total number of UGTs within S. rueppellii compared to other Dipteran species. For example, in the Drosophila melanogaster genome, expansion is only seen in 3 UGT families (UGT35, UGT303, UGT37); and in three mosquito species (Anopheles sinensis, Anopheles gambiae, Aedes aegypti) expansion is only seen in UGT308 [94]. S. rueppellii also has a much higher number of UGT genes compared to other pollinator species.

Hemiptera crop pest species tend to have higher numbers of UGT genes than Diptera, as shown in Table 6 . This tends to be the result of substantial gene expansion concentrated within a single UGT family. For example: a UGT352 expansion in Bemisia tabaci accounted for 36 of its 76 UGTs; the UGT344 family accounted for 35 of Acyrthosiphon pisum's 72 UGTs and the UGT201 family accounted for 33 of Tetranychus urticae's 81 UGTs. These lineage specific expansions have previously been linked to increased detoxification of plant secondary metabolites [100] and therefore the increased number of UGTs in Hemiptera compared to Diptera may be linked to differences in diet. Host plant adaptation alone has been shown to usually be insufficient to confer insecticide resistance, and therefore higher numbers of UGTs in Hemiptera 
cannot be assumed to correspond to increased insecticide tolerance/resistance [101]. However, upregulation of UGTs from 7 different UGT families, including 6 UGT344 members, has been associated with thiamethoxam resistance in Aphis gossypii [102]. It is therefore possible that expansion in UGT families may be associated with both host plant adaptation and insecticide resistance. Further study into the role of individual UGTs would be needed to clarify whether differences in total numbers of UGTs are associated with differences in insecticide tolerance/resistance between Hemiptera and Diptera.

9 of the S. rueppellii UGT genes belong to the UGT302 and UGT308 families, which are suggested to be the ones most associated with resistance to pyrethroid insecticides [94]. This suggests that expansion of these families in $S$. rueppellii could be a response to pyrethroid exposure. Expansion of these gene families has been reported in A. sinensis - 14 of its 30 UGT genes belonged to the UGT302/308 families and 7 of these were considered strong candidates for pyrethroid resistance [94].

The most significant expansion for $S$. rueppellii is seen in the UGT431 family. This family is unique to $S$. rueppellii, but is closest in sequence similarity to the UGT37 and UGT430 families which also exhibited some expansion. The UGT37 family has been shown to be upregulated during organophosphorus pesticide exposure in Caenorhabditis elegans [103]. The UGT37 family exhibits lineage specific expansion in D. melanogaster and is its largest UGT gene family with members spread across five different genome locations [93]. This differs from the $S$. rueppellii genome, where the majority (12/14) of the UGT37 and UGT431 families are located in a cluster of adjacent genes on chromosome 2 within $0.17 \mathrm{Mb}$ of genomic space. This could suggest the UGT37 family may have expanded more recently in $S$. rueppellii, however, the percentage identity within this cluster ranges from $33-70 \%$, which indicates that at least part of the cluster can be considered "old". Since these genes have not been fully dispersed in the genome, there may be a selective advantage for preserving the cluster on chromosome 2 as a heritable unit, i.e. UGT37/431 members may be required for the same mechanism. Based on the links of UGT37 to pesticide resistance, the expansion of the UGT37/431 families and preservation of the gene cluster could be an adaptational response to pesticide exposure.

\section{Glutathione S-transferases}

The glutathione $S$-transferases (GSTs) family is large and functionally diverse, and has been shown to confer resistance to all main insecticide classes. For example, the delta and epsilon classes have been linked to pyrethroid resistance in $A$. aegypti and N. lugens $[104,105]$. GST-mediated detoxification of insecticides takes place via several different mechanisms, including protecting against oxidative stress, binding and sequestration of the insecticide and by catalysing the conjugation of glutathione to insecticides and their metabolites to reduce their toxicity and facilitate excretion, respectively [39].

S. rueppellii has 23 GSTs (Table 7), which are located on proposed chromosomes 1-3, with members of the same family located on the same chromosome. (Chr1: Theta and Omega, Chr2: Epsilon, Chr3: Sigma, Delta and Zeta.) A phylogenetic tree of these GSTs, with likely tandem duplications indicated, is shown in figure 6 . The total number of GSTs is slightly lower in S. rueppellii compared to other Diptera species, although higher than other pollinators.

Sigma-GSTs are associated with detoxification of oxidants produced during pollen and nectar metabolism in bees [106]. However, S. rueppellii has a reduced number of sigma-GSTs compared to other pollinators. This suggests $S$. rueppellii may use different detoxification enzymes to cope with these oxidants, or perhaps a different pathway for pollen and nectar metabolism. 
Table 7

Numbers of GST genes annotated in Sphaerophoria rueppellii (this study), Drosophila melanogaster [107], Aedes aegypti [108], Anopheles gambiae [109], Culex pipiens quinquefasciatus [110], Apis mellifera, Bombus impatiens, Bombus huntii [111], Thrips palmi [99], Myzus persicae, Acyrthosiphon pisum, Trialeurodes vaporariorum and Bemisia tabaci [112] and their distribution across classes.

\begin{tabular}{|c|c|c|c|c|c|c|c|c|c|c|c|c|c|}
\hline & \multicolumn{5}{|c|}{ S. rueppellii + close relatives } & \multicolumn{3}{|c|}{ Pollinators } & \multicolumn{5}{|c|}{ Crop pests } \\
\hline & $\mathrm{Sr}$ & Dm & $\mathrm{Aa}$ & $\mathrm{Ag}$ & Cp & Am & $\mathrm{Bi}$ & $\mathrm{Bh}$ & $\mathrm{Tp}$ & Mp & Ap & Tv & Bt \\
\hline Delta & 4 & 9 & 8 & 12 & 14 & 1 & - & - & 14 & 3 & 11 & 9 & 14 \\
\hline Epsilon & 11 & 14 & 8 & 8 & 10 & 0 & - & 0 & 0 & 0 & 0 & 1 & 0 \\
\hline Omega & 3 & 4 & 1 & 1 & 1 & 1 & - & - & 1 & 1 & 1 & 0 & 1 \\
\hline Sigma & 1 & 1 & 1 & 1 & 1 & 4 & - & 3 & 6 & 12 & 5 & 3 & 6 \\
\hline Theta & 3 & 4 & 4 & 2 & 6 & 1 & - & - & 1 & 1 & 2 & 0 & 0 \\
\hline Zeta & 1 & 2 & 1 & 1 & 0 & 1 & - & - & 2 & 0 & 0 & 2 & 2 \\
\hline Microsomal & 0 & 3 & 3 & 3 & 3 & 2 & - & - & 1 & 2 & 2 & 3 & 2 \\
\hline Total & 23 & 37 & 26 & 28 & 35 & 10 & 15 & 11 & 25 & 19 & 21 & 18 & 25 \\
\hline
\end{tabular}

Within the Diptera species the majority of GSTs are present within the epsilon and delta class, however, for $S$. rueppellii whilst the numbers of epsilon GSTs are comparable to other Diptera species, the numbers of delta class GSTs are notably lower.

The epsilon class is the largest class in S. rueppellii, as a result of substantial class-specific expansion. 7 epsilon members are clustered within $31 \mathrm{~kb}$, with a percentage identity ranging from $35-81 \%$, this indicates that whilst some members of the cluster are the result of recent tandem duplications, others are the result of far older duplications. Clusters of epsilon GSTs are common across Diptera species, with clusters of 8 epsilon genes seen in A. aegypti and A. gambiae and a cluster of 11 epsilon genes in $D$. melanogaster. The preservation of these clusters suggests that maintaining epsilon genes as a heritable cluster confers a selective advantage, likely in terms of conferring increased insecticide resistance. This cluster and class specific expansion may therefore imply an increased degree of GST delta-linked pyrethroid tolerance/resistance in S. rueppellii compared to Hemiptera crop pests, which have at most 1 epsilon gene.

In contrast to the epsilon class, S. rueppellii's delta class is far smaller, as a result of minimal recent class-specific expansion. Only 2 of the genes are directly adjacent, and were likely a recent tandem duplication based on their $88 \%$ sequence identity, whilst the other two members are dispersed through the genome (across 7.8Mb of genomic space). This follows the pattern seen in some other Diptera species, which also have delta genes more widely dispersed than epsilon. For example, 3 separate clusters are seen in both $A$. aegypti and A. gambiae, (although in D. melanogastera single cluster of 11 delta genes is present) [113]. This reduced number of delta GSTs could imply a reduced degree of GST delta-linked pyrethroid resistance in $S$. rueppellii compared to Hemiptera crop pests, although this may be counteracted by the significant expansion within the epsilon class. The lack of preservation of delta clusters may also suggest that they confer a less significant selective advantage than do the epsilon GSTs.

The sigma class of GSTs has been associated with the detoxification of organophosphorus insecticides [114]. All Diptera species included in analysis had only 1 sigma gene, and this was also the case for $S$. rueppellii. All crop pest species had larger sigma classes. This may imply a reduced level of GST sigma-linked organophosphorus resistance compared to Hemiptera crop pests. 


\section{Carboxyl/choline esterases}

Carboxyl/choline esterases (CCEs) are associated with insecticide resistance, notably to organophosphates, and to a lesser degree carbamates and pyrethroids [115]. For example esterase-based organophosphate resistance has been reported in three Culex species [116] and synergist bioassays have shown that esterases are responsible for metabolic resistance to pyrethroids (deltamethrin) and organophosphates (temephos) in A. aegypti [117].

Table 8

Numbers of CCEs annotated in Sphaerophoria rueppellii (this study), Drosophila melanogaster, Aedes aegypti, Anopheles gambiae [118], Culex pipiens quinquefasciatus [110], Apis mellifera, Bombus impatiens, Bombus huntii [111], Frankliniella occidentalis [119], Myzus persicae [120], Acyrthosiphon pisum, Bemisia tabaci [121] and Trialeurodes vaporariorum [122] and their distribution across classes and clades.

\begin{tabular}{|c|c|c|c|c|c|c|c|c|c|c|c|c|c|c|}
\hline & & \multicolumn{4}{|c|}{$\begin{array}{l}\text { S. rueppellii and close } \\
\text { relatives }\end{array}$} & \multicolumn{4}{|c|}{ Pollinators } & \multicolumn{5}{|c|}{ Crop pests } \\
\hline & & $\mathrm{Sr}$ & Dm & Cp & $\mathrm{Aa}$ & $\mathrm{Ag}$ & Am & $\mathrm{Bi}$ & $\mathrm{Bh}$ & Fo & Mp & Ap & Tv & Bt \\
\hline \multicolumn{2}{|l|}{ Dietary class } & 15 & 13 & 30 & 22 & 16 & 8 & - & - & 28 & 5 & 5 & 12 & 6 \\
\hline \multicolumn{2}{|c|}{$\begin{array}{l}\text { Hormone/semiochemical processing } \\
\text { class }\end{array}$} & 13 & 8 & 26 & 15 & 14 & 5 & - & - & 7 & 12 & 16 & 6 & 19 \\
\hline \multirow{7}{*}{$\begin{array}{l}\text { Neuro- } \\
\text { developmental } \\
\text { class }\end{array}$} & Glutactins & 4 & 5 & 6 & 7 & 10 & 0 & - & - & 2 & 0 & 0 & 1 & 1 \\
\hline & AChE & 1 & 1 & 1 & 2 & 2 & 2 & - & - & 2 & 3 & 2 & 2 & 4 \\
\hline & uncharacterised & - & 1 & 2 & 1 & 1 & 3 & - & - & 2 & 1 & 1 & 1 & 1 \\
\hline & gliotactin & 1 & 1 & 1 & 1 & 1 & 1 & - & - & 1 & 1 & 1 & 1 & 1 \\
\hline & neuroligin & 5 & 4 & 3 & 5 & 5 & 5 & - & - & 7 & 0 & 3 & 3 & 10 \\
\hline & neurotactin & 1 & 2 & 2 & 2 & 2 & - & - & - & 1 & 0 & 0 & 1 & 0 \\
\hline & Subtotal & 12 & 14 & 15 & 18 & 21 & 11 & - & - & 15 & 5 & 7 & 9 & 17 \\
\hline \multicolumn{2}{|l|}{ Total } & 40 & 35 & 71 & 55 & 51 & 24 & 22 & 23 & 50 & 22 & 28 & 27 & 42 \\
\hline
\end{tabular}

S. rueppellii has 40 full-length carboxylesterase genes (Table 8) which are distributed across proposed chromosomes 1-5 with 19 of the genes arranged in 7 clusters of 2-4 genes (figure 7). The total number of CCEs for $S$. rueppellii and the distribution of genes across the 3 main classes is comparable to other Diptera species. The numbers and distribution of CCEs is also similar between Diptera and Hemiptera, with the only noticeable differences being a lower average number of 'dietary' esterases in Hemiptera species and a higher number of 'glutactins' in Diptera. Compared to other pollinators, $S$. rueppellii has a much higher number of CCE genes.

The so-called 'dietary' class of CCEs has been shown to be involved in insecticide and xenobiotic detoxification [118] and amplification of genes within this class, i.e. esterase E4/B1-like genes, has been linked to organophosphate resistance in hemipteran and dipteran species (M persicae, N. Iugens, S. graminum and Culex mosquitoes) [116, 123-127]. Within the S. rueppellii genome, multiple clusters of high similarity, adjacent esterase E4/B1 genes indicate recent tandem duplications, which could confer some tolerance/resistance to organophosphorus insecticides. In cases where the number of dietary genes in S. rueppellii is higher than Hemiptera crop pests there could be an increased degree of organophosphate resistance. 


\section{ATP-binding cassette transporters}

ATP-binding cassette transporters (ABCs) are the largest known group of active transporters and are able to eliminate by translocation xenobiotic compounds such as secondary metabolites produced by plants or insecticides [128]. The ABC transporters are subdivided into eight subfamilies (ABCA-H), of which $A B C B, A B C C$ and $A B C G$ are the most associated with resistance to a variety of insecticides including pyrethroids, carbamates, organophosphates and neonicotinoids [129].

Table 9

Numbers of ABC transporter genes annotated in Sphaerophoria rueppellii (this study), Drosophila melanogaster [129], Bactrocera dorsalis [130], Anopheles gambiae, Culex pipiens quinquefasciatus [131], Apis mellifera [132], Aedes aegypti

[133], Anopheles sinensis [134], Frankliniella occidentalis [119], Thrips palmi [99], Aphis gossypii [135], Trialeurodes vaporariorum [136] Diuraphis noxia and Bemisia tabaci [137] and their distribution across subfamilies.

\begin{tabular}{|c|c|c|c|c|c|c|c|c|c|c|c|c|c|c|}
\hline & \multicolumn{7}{|c|}{ S. rueppellii + close relatives } & \multirow{2}{*}{$\begin{array}{l}\text { Pollinator } \\
\text { Am }\end{array}$} & \multicolumn{6}{|c|}{ Crop pests } \\
\hline & $\mathrm{Sr}$ & Dm & Bd & Aga & $\mathrm{Aa}$ & As & Cp & & Fo & Tp & Dn & Ago & Tv & $\mathrm{Bt}$ \\
\hline $\mathrm{ABCA}$ & $\begin{array}{l}11 \\
\left(12^{\star}\right)\end{array}$ & 10 & 7 & 8 & 10 & 10 & 9 & 3 & 3 & 3 & 3 & 4 & 3 & 8 \\
\hline$A B C B$ & $6\left(7^{*}\right)$ & 8 & 7 & 5 & 5 & 5 & 5 & 5 & 5 & 4 & 6 & 5 & 9 & 3 \\
\hline$A B C C$ & 8 & 14 & 9 & 15 & 15 & 16 & 18 & 9 & 19 & 12 & 24 & 25 & 7 & 6 \\
\hline$A B C D$ & 3 & 2 & 2 & 2 & 2 & 2 & 2 & 2 & 2 & 2 & 3 & 2 & 4 & 2 \\
\hline$A B C E$ & 1 & 1 & 1 & 2 & 1 & 1 & 2 & 1 & 1 & 2 & 1 & 1 & 1 & 1 \\
\hline $\mathrm{ABCF}$ & 3 & 3 & 3 & 3 & 3 & 3 & 3 & 3 & 3 & 3 & 3 & 4 & 3 & 3 \\
\hline ABCG & 10 & 15 & 15 & 17 & 15 & 21 & 28 & 15 & 22 & 16 & 26 & 30 & 9 & 23 \\
\hline $\mathrm{ABCH}$ & 3 & 3 & 3 & 3 & 4 & 3 & 3 & 3 & 13 & 7 & 11 & 0 & 9 & 9 \\
\hline Total & $\begin{array}{l}45 \\
(47 *)\end{array}$ & 56 & 47 & 55 & $\begin{array}{l}53 \text { (62 with } 9 \text { in } \\
\text { ABCJ) }\end{array}$ & 61 & 70 & 41 & 70 & 49 & 77 & 71 & 45 & 55 \\
\hline
\end{tabular}

S. rueppellii has 47 ABC genes (Table 9), which are distributed across proposed chromosomes 1-6, with 3 of the genes located on scaffolds external to the chromosome superscaffolds. 20 of the genes are located in 9 clusters of 2-3 (figure 8). The total number of $A B C$ genes in $S$. rueppellii is at the lower end of that seen for other Diptera species as well as for Hemiptera crop pests, but slightly higher than another species of pollinator.

The distribution of $S$. rueppellis ABC genes across subfamilies is similar to other species, except for the ABCC and ABCG subfamilies, which are smaller in $S$. rueppellii than all other Diptera species and the majority of Hemiptera crop pests. These are two of the families most associated with insecticide resistance, and so their reduced size suggests that ABCmediated tolerance/resistance to insecticides could be lower in S. rueppellii compared to these other species.

The ABCA subfamily is expanded in Diptera, whilst the ABCH subfamily is expanded in Hemiptera. However these subfamilies do not have strong links to insecticide resistance, and so these differences would likely not contribute to any variation in tolerance/resistance levels.

The percentage identity of $A B C$ genes within S. rueppellii ranges from $0 \%-71 \%$, with the exception of one pair of genes with an identity of $89 \%$. This suggests that there has been little recent lineage specific expansion within the S. rueppellii ABC 
transporter family, and this is supported by the numbers of the genes in the ABC subfamilies, which are either similar to or lower than other Diptera species. Any lineage-specific expansion seen in S. rueppellii is minimal, demonstrated by the small size of gene clusters. Species-specific and lineage-specific ABC expansions on a much larger scale have been reported in a variety of arthropods such as Tribolium castaneum and Tetranychus urticae, although whether these expansions contribute directly to increased insecticide resistance is not yet known [129]

\section{Cytochrome P450 monooxygenases}

Cytochrome P450 monooxygenases (P450s) are a diverse superfamily capable of metabolizing a huge variety of endogenous and exogenous substrates. In insects they are associated with growth and development, metabolism of pesticides and plant toxins as well as the production and metabolism of insect hormones and pheromones [138, 139]. $\mathrm{P} 450$ s are associated with the resistance to insecticides from a variety of classes, including pyrethroids, carbamates and neonicotinoids and many examples of resistance are linked to upregulated P450s [140-143]. They are also linked to the activation of organophosphates and other pro-insecticides (a form of insecticide which is metabolized into an active form inside the host) [144] often as a result of downregulation [145, 146].

Table 10

Total numbers of Cytochrome P450 genes annotated in Sphaerophoria rueppellii (this study), Musca domestica, Drosophila melanogaster [147], Anopheles gambiae, Aedes aegypti [148], Culex pipiens quinquefasciatus [110], Apis mellifera [149], Bombus impatiens, Bombus huntii [95], Frankliniella occidentalis, Thrips palmi [119], Myzus persicae, Acyrthosiphon pisum [120], Trialeurodes vaporariorum [136] and Bemisia tabaci [150].

\begin{tabular}{|c|c|c|c|c|c|c|c|c|c|c|c|c|c|c|c|}
\hline & \multicolumn{6}{|c|}{ S. rueppellii + close relatives } & \multicolumn{3}{|c|}{ Pollinators } & \multicolumn{6}{|c|}{ Crop pests } \\
\hline & $\mathrm{Sr}$ & Md & Dm & $\mathrm{Ag}$ & $\mathrm{Aa}$ & Cp & Am & $\mathrm{Bi}$ & $\mathrm{Bh}$ & Fo & Tp & $\mathrm{Mp}$ & Ap & Tv & $\mathrm{Bt}$ \\
\hline CYP2 & 6 & 8 & 7 & 10 & 11 & 14 & 8 & - & - & 12 & 12 & 3 & 10 & 7 & 18 \\
\hline CYP3 & $34(37)$ * & 65 & 35 & 41 & 80 & 88 & 31 & - & - & 22 & 26 & 63 & 33 & 41 & 76 \\
\hline CYP6 & 22 & 46 & 22 & - & - & - & - & - & - & 18 & - & - & 29 & 34 & 47 \\
\hline CYP9 & 2 & 7 & 5 & - & - & - & - & - & - & 0 & - & - & 0 & 0 & 0 \\
\hline Other & 10 & 12 & 8 & - & - & - & - & - & - & 4 & - & - & 4 & 7 & - \\
\hline CYP4 & $15(16)^{*}$ & 55 & 33 & 45 & 58 & 83 & 4 & 5 & 2 & 37 & 42 & 48 & 32 & 25 & 73 \\
\hline Mitochondrial & 14 & 18 & 11 & 9 & 9 & 11 & 6 & - & - & 10 & 11 & 1 & 8 & 7 & 4 \\
\hline Total & $69(73) *$ & 146 & 86 & 105 & 158 & 196 & 49 & 49 & 44 & 81 & 91 & 115 & 83 & 80 & 171 \\
\hline
\end{tabular}

A total of 69 full-length P450 genes were identified in the $S$. rueppellii genome, as well as 4 P450 fragment genes (Table 10). These genes were named by Dr David Nelson using his in-house pipeline [82]. The total number of P450s varies widely between insect species, ranging from 44 for Bombus huntii to 196 for $C$. pipiens. S. rueppellii falls at the lower end of this range, however when compared to other dipteran species, this is mainly due to the reduced size of the CYP4 clan.

34 of the P450 genes have 55-97\% identity to another sequenced P450, 38 have $40-55 \%$ identity, and 1 gene has $<40 \%$ identity. 9 genes (CYP18A1, CYP301-304A1, CYP307A2, CYP314A1, CYP315A1 and CYP49A1) were classified as 
orthologs to P450s from Lucilia cuprina, Ceratitis capitata and Musca domestica. These genes are involved in a conserved pathway, found in all insects, for the essential growth hormone 20-hydroxyecdysone [151]. Orthologs were not present for other genes, likely because other P450s are involved in detoxification, and therefore vary during evolution based on the organism's environment and adaptation.

The CYPome diversity value was $52 \%$, based on the presence of 38 CYP subfamilies and 73 genes. The CYPome follows the pattern of other arthropods, with most CYP families having few genes, whilst only a few CYP families have many genes. [149]

The majority of S. rueppellii P450s (34) belong to the CYP3 clan (Table 10), which is the one most associated with insecticide resistance, notably the CYP6 and CYP9 families [139], both of which were present in S. rueppellii. CYP3 is also the largest clan in other pollinators and in several other diptera species and hemipteran crop pest species.

The largest sub-family specific expansion is in clan 3, within the CYP6Zx family, with 16 members: CYP6ZQ1-11, CYP6ZR1-4 and CYPZS1 (figure 9). CYP6ZQ1-11 (excluding Q7) are located contiguously within a 0.2Mb region of chromosome 3 (figure 10). Within this cluster there is no consistent relationship or pattern between the proximity of the CYP6Zx genes or their gene structure with their percent identity, which ranged from 33-90\%. The lower end of the percent identity within the cluster indicates that at least part of the cluster can be considered "old", and therefore, since these genes have not been fully dispersed in the genome, there may be a selective advantage for preserving the cluster on chromosome 3 as a heritable unit.

Whether the large CYP6Zx expansion may confer an increased degree of tolerance to xenobiotics in S. rueppellii remains to be investigated. Overall, numbers of the resistance-associated CYP3 clan are similar or lower than Hemiptera crop pests, suggesting that P450-mediated insecticide tolerance/resistance mechanisms may not be as prevalent as for other species.

The CYP4 clan is vastly expanded in many arthropods [152], and whilst the CYP4 clan is not as strongly associated with insecticide resistance as CYP3, studies have shown upregulation of some CYP4 genes in response to insecticide exposure [141, 153-155]. S. rueppellii has a lower number of CYP4 genes compared to many other dipteran species and crop pests, however, compared to other pollinators the CYP4 subfamily is relatively large. A reduced number of CYP4 genes is common within pollinators $[95,156]$, but the reasons behind this are not yet known.

Pollinators use P450s for the detoxification of pollen flavonoids, notably the CYP6AS subfamily which is often expanded in honey bees; however, this subfamily is absent in $S$. rueppellii $[157,158]$. It is likely that another subfamily is responsible for flavonoid detoxification in S. rueppellii (possibly the expanded CYP6Zx subfamily) and future studies assessing P450 upregulation in response to flavonoids could help identify this.

\section{Point Mutations}

Point mutations in genes encoding insecticide targets which are known to confer insensitivity to insecticides were searched for in the $S$. rueppellii genes. This includes those in the sodium channel para gene, which can confer resistance to pyrethroids; the GABA-gated ion channel RDL which can lead to multiple insecticide resistance; the acetylcholinesterase (ace-2) enzyme which is associated with organophosphate and carbamate resistance; the Ryanodine receptor which is linked to diamide resistance and acetyl CoA carboxylase which is linked to keto-enol resistance. Despite mutations in these genes having been observed across Diptera species including house flies and mosquitoes as well as crop pests such as whiteflies, aphids and diamondback moths, none were found in this S. rueppellii genome [159-173]. 
Overall, target site mediated tolerance/resistance is not seen in $S$. rueppellii. Although it is important to note that the $S$. rueppellii genome assembly was a consensus of $\sim 30$ individuals, therefore mutations would likely only be apparent if they were present in the majority of the population.

\section{Conclusions}

Here we present the first high quality genome of S. rueppellii including the mitochondrial genome enabled by PacBio longread technology combined with low error-rate short-read Illumina sequencing. Hi-C data permitted further scaffolding of this genome to a near-chromosome level assembly. The genome completeness is of excellent quality for comparative and functional genomics analyses and provides a useful first reference for predatory Syrphidae.

Comparative analyses of $S$. rueppellii with crop pests showed evidence that $S$. rueppellii has a detoxification gene inventory comparable to selected crop pests, with a few notable differences: lineage-specific expansions were seen within detoxification gene families such as UGTs and P450, whereas the ABC transporter family lacks such expansions compared to some crop pests. No mutations were found in common insecticide target-sites, suggesting a lack of selectivity of insecticides at the protein/receptor binding level.

Comparative analyses of $S$. rueppellii with pollinators showed that $S$. rueppellii has an increased number of genes in all detoxification families, in particular: UGTs, non-sigma class GSTs and CYP4 P450s. This could be in part due to $S$. rueppellii needing more detoxification genes for its diet: hoverflies lack the eusocial behavioural mechanisms seen in bees, such as processing nectar into honey and converting pollen into 'beebread', which result in a dilution of toxins and hence reduce the need for detoxification enzymes in bees [156]. Additionally, the considerably longer migratory distance covered by hoverflies compared to bees [21] may have resulted in hoverflies being exposed to a wider variety of xenobiotics, and could perhaps have resulted in expansion of associated detoxification genes.

Despite the reduced number of detoxification genes in pollinators such as $A$. mellifera, they appear to be no more sensitive to insecticides than other insects $[156,174]$. Insects with a pollen-based diet have been found to have an increased degree of insecticide tolerance, with many of the same genes being upregulated in response to both pollen and to certain insecticides [175]. This suggests that the unique set of detoxification genes required by pollinators for their diet, could perhaps impart an increased degree of insecticide tolerance without the need for the extent of gene expansion seen in other insect species. This may mean that despite $S$. rueppellii having fewer detoxification genes than some crop pests, this might not necessarily be indicative of reduced insecticide tolerance. However, this is not to say that insecticides are not a major problem for $S$. rueppellii, with clear evidence that the same neonicotinoids (imidacloprid and thiamethoxam) which are toxic to honey bees are also toxic to $S$. rueppellii $[176,177]$.

This study provides a good basis for beginning to identify differences in genes encoding potential tolerance/resistance mechanisms between crop pests and $S$. rueppellii which could be exploited when selecting targeted insecticides for use in IPM strategies. Evidence of gene expansions in resistance-associated gene families implies that $S$. rueppellii is certainly capable of developing resistance to a variety of insecticides, which could be used to our advantage through the selective breeding and selection of resistant strains of $S$. rueppellii for use in IPM.

An interesting future comparison could be to look at the differences in olfactory genes between $S$. rueppellii and $E$. pertinax (the non-predatory European hoverfly), as this may give some indication of the genes involved in detecting aphid pheromones and avoidance of parasitised aphids.

\section{Declarations}

\section{Ethics approval and consent to participate}

Page $18 / 37$ 
Not applicable.

\section{Consent for publication}

Not applicable.

\section{Availability of data and materials}

The genome and transcriptome assemblies generated in this study (as well as the raw sequencing data used to produce them) are available under BioProject: PRJEB48036. The manually curated Sphaerophoria rueppellii genes used for comparative analyses are included in additional file 1.

\section{Competing interests}

The authors declare that they have no competing interests.

\section{Funding}

This research was funded by the Pest Genomics Initiative, a collaborative project between Rothamsted Research, Bayer Crop Science and Syngenta AG. Rothamsted Research gratefully acknowledges strategic funding from the UK Biotechnology and Biological and Sciences Research Council (UKRI-BBSRC). The content is solely the responsibility of the authors and does not necessarily reflect the official views of the funding bodies.

\section{Authors' contributions}

The Pest Genomics Initiative (BB, CR, EG, KH-P, LF, RK and RN) devised the original conceptual ideas. EB performed the DNA and RNA extractions with help from MW. EB assembled and annotated the genome with guidance from RK and DH. EB performed the comparative analyses. FM, RK and KH-P supervised the project. EB wrote the manuscript. All authors read, edited and approved the final manuscript.

\section{Acknowledgements}

Acknowledgements go to Dr. David R. Nelson of the International Committee on the Nomenclature for Cytochrome P450 Enzymes for classification of Sphaerophoria rueppellii P450s and to Dr. Michael H. Court of the UGT Nomenclature Committee for classification of Sphaerophoria rueppellii UGTs.

\section{References}

1. Oerke E-C. Crop losses to pests. J Agric Sci 2006,144:31-43.

2. Geiger F, Bengtsson J, Berendse F, Weisser WW, Emmerson M, Morales MB, et al. Persistent negative effects of pesticides on biodiversity and biological control potential on European farmland. Basic Appl Ecol 2010,11:97-105.

3. Bottrell DG, Schoenly KG. Resurrecting the ghost of green revolutions past: The brown planthopper as a recurring threat to high-yielding rice production in tropical Asia. J Asia Pac Entomol 2012,15:122-40.

4. Debach P, Rosen D. Biological control by natural enemies (second edition). J Trop Ecol 1992,8:216-216.

5. Rojo S, coaut GF, coaut M-GM, coaut NJ, coaut MM. A world review of predatory hoverflies (Diptera, Syrphidae: Syrphinae) and their prey. sidalc.net, 2003.

6. Wotton KR, Gao B, Menz MHM, Morris RKA, Ball SG, Lim KS, et al. Mass Seasonal Migrations of Hoverflies Provide Extensive Pollination and Crop Protection Services. Curr Biol 2019,29:2167-73.e5. 
7. Ramsden M, Menendez R, Leather S, Wäckers F. Do natural enemies really make a difference? Field scale impacts of parasitoid wasps and hoverfly larvae on cereal aphid populations. Agric For Entomol 2017,19:139-45.

8. Tenhumberg B, Poehling H-M. Syrphids as natural enemies of cereal aphids in Germany: Aspects of their biology and efficacy in different years and regions. Agric Ecosyst Environ 1995,52:39-43.

9. Tenhumberg B. Predicting predation efficiency of biocontrol agents: linking behavior of individuals and population dynamics. International Congress on Environmental Modelling and Software, scholarsarchive.byu.edu, 2004.

10. ROTHERAY, GE. Colour guide to hoverfly larvae (Diptera, Syrphidae). Dipter Dig 1993,9:1-155.

11. Dunn L, Lequerica M, Reid CR, Latty T. Dual ecosystem services of syrphid flies (Diptera: Syrphidae): pollinators and biological control agents. Pest Manag Sci 2020,76:1973-9.

12. Hopper JV, Nelson EH, Daane KM, Mills NJ. Growth, development and consumption by four syrphid species associated with the lettuce aphid, Nasonovia ribisnigri, in California. Biol Control 2011,58:271-6.

13. Pekas A, De Craecker I, Boonen S, Wäckers FL, Moerkens R. One stone, two birds: concurrent pest control and pollination services provided by aphidophagous hoverflies. Biol Control 2020,149:104328.

14. Mizuno M, Itioka T, Tatematsu Y, Itô Y. Food utilization of aphidophagous hoverfly larvae (Diptera: Syrphidae, Chamaemyiidae) on herbaceous plants in an urban habitat. Ecol Res 1997,12:239-48.

15. Dib H, Simon S, Sauphanor B, Capowiez Y. The role of natural enemies on the population dynamics of the rosy apple aphid, Dysaphis plantaginea Passerini (Hemiptera: Aphididae) in organic apple orchards in south-eastern France. Biol Control 2010,55:97-109.

16. Messelink GJ, Janssen A. Increased control of thrips and aphids in greenhouses with two species of generalist predatory bugs involved in intraguild predation. Biol Control 2014,79:1-7.

17. Freier B, Triltsch H, Möwes M, Moll E. The potential of predators in natural control of aphids in wheat: Results of a tenyear field study in two German landscapes. Biocontrol 2007,52:775-88.

18. Brewer MJ, Elliott NC. Biological control of cereal aphids in north america and mediating effects of host plant and habitat manipulations. Annu Rev Entomol 2004,49:219-42.

19. Rotheray GE, Gilbert F. The natural history of hoverflies. Forrest text, 2011.

20. Rader R, Cunningham SA, Howlett BG, Inouye DW. Non-Bee Insects as Visitors and Pollinators of Crops: Biology, Ecology, and Management. Annu Rev Entomol 2020,65:391-407.

21. Doyle T, Hawkes WLS, Massy R, Powney GD, Menz MHM, Wotton KR. Pollination by hoverflies in the Anthropocene. Proc Biol Sci 2020,287:20200508.

22. Hodgkiss D, Brown MJF, Fountain MT. The effect of within-crop floral resources on pollination, aphid control and fruit quality in commercial strawberry. Agric Ecosyst Environ 2019,275:112-22.

23. Jauker F, Wolters V. Hover flies are efficient pollinators of oilseed rape. Oecologia 2008,156:819-23.

24. Thompson FC, Rotheray GE, Zumbado MA, Brown BV, Borkent A, Cumming JM, et al. Manual of Central American Diptera 2010.

25. Darwin Tree of Life - Reading the genomes of all life: a new platform for understanding our biodiversity n.d. https://www.darwintreeoflife.org/ (accessed July 22, 2021).

26. Hoy MA, Waterhouse RM, Wu K, Estep AS, loannidis P, Palmer WJ, et al. Genome Sequencing of the Phytoseiid Predatory Mite Metaseiulus occidentalis Reveals Completely Atomized Hox Genes and Superdynamic Intron Evolution. Genome Biol Evol 2016,8:1762-75.

27. Werren JH, Richards S, Desjardins CA, Niehuis O, Gadau J, Colbourne JK, et al. Functional and Evolutionary Insights from the Genomes of Three Parasitoid Nasonia Species. Science 2010,327:343-8.

28. Bailey E, Field L, Rawlings C, King R, Mohareb F, Pak K-H, et al. A scaffold-level genome assembly of the pirate bug, Orius laevigatus, and a comparative analysis of insecticide resistance-related gene families with hemipteran crop 
pests. Research Square 2021. https://doi.org/10.21203/rs.3.rs-537204/v1.

29. Ando T, Matsuda T, Goto K, Hara K, Ito A, Hirata J, et al. Repeated inversions within a pannier intron drive diversification of intraspecific colour patterns of ladybird beetles. Nat Commun 2018,9:1-13.

30. European Commission. Directive 2009/128/EC on the sustainable use of pesticides. Official Journal of the European Union, 2009. https://doi.org/10.2861/78.

31. Cameron PJ, Walker GP, Hodson AJ, Kale AJ, Herman TJB. Trends in IPM and insecticide use in processing tomatoes in New Zealand. Crop Prot 2009,28:421-7.

32. Kranthi KR, Russell DA. Changing Trends in Cotton Pest Management. In: Peshin R, Dhawan AK, editors. Integrated Pest Management: Innovation-Development Process: Volume 1, Dordrecht: Springer Netherlands, 2009, p. 499-541.

33. Meissle M, Mouron P, Musa T, Bigler F, Pons X, Vasileiadis VP, et al. Pests, pesticide use and alternative options in European maize production: current status and future prospects. J Appl Entomol 2009,134:357-75.

34. Hillocks RJ. Farming with fewer pesticides: EU pesticide review and resulting challenges for UK agriculture. Crop Prot 2012,31:85-93.

35. Lechenet M, Dessaint F, Py G, Makowski D, Munier-Jolain N. Reducing pesticide use while preserving crop productivity and profitability on arable farms. Nature Plants 2017,3:1-6.

36. Heckel DG. Insecticide Resistance After Silent Spring. Science 2012,337:1612-4.

37. Li X, Shi H, Gao X, Liang P. Characterization of UDP-glucuronosyltransferase genes and their possible roles in multiinsecticide resistance in Plutella xylostella (L.). Pest Manag Sci 2018,74:695-704.

38. Merzendorfer H. Chapter One - ABC Transporters and Their Role in Protecting Insects from Pesticides and Their Metabolites. In: Cohen E, editor. Advances in Insect Physiology, vol. 46, Academic Press, 2014, p. 1-72.

39. Pavlidi N, Vontas J, Van Leeuwen T. The role of glutathione S-transferases (GSTs) in insecticide resistance in crop pests and disease vectors. Curr Opin Insect Sci 2018,27:97-102.

40. Scott JG. Cytochromes P450 and insecticide resistance. Insect Biochem Mol Biol 1999,29:757-77.

41. Sogorb MA, Vilanova E. Enzymes involved in the detoxification of organophosphorus, carbamate and pyrethroid insecticides through hydrolysis. Toxicol Lett 2002,128:215-28.

42. Rane RV, Ghodke AB, Hoffmann AA, Edwards OR, Walsh TK, Oakeshott JG. Detoxifying enzyme complements and host use phenotypes in 160 insect species. Current Opinion in Insect Science 2019,31:131-8.

43. Roderick GK, Navajas M. Genes in new environments: genetics and evolution in biological control. Nat Rev Genet 2003,4:889-99.

44. Marçais G, Kingsford C. A fast, lock-free approach for efficient parallel counting of occurrences of k-mers. Bioinformatics 2011,27:764-70.

45. Rhyker Ranallo-Benavidez T, Jaron KS, Schatz MC. GenomeScope 2.0 and Smudgeplot for reference-free profiling of polyploid genomes. Nat Commun 2020,11:1-10.

46. Bradnam K. A script to calculate a basic set of metrics from a genome assembly 2011. http://korflab.ucdavis.edu/Datasets/Assemblathon/Assemblathon2/Basic_metrics/assemblathon_stats.pl.

47. Simão FA, Waterhouse RM, loannidis P, Kriventseva EV, Zdobnov EM. BUSCO: assessing genome assembly and annotation completeness with single-copy orthologs. Bioinformatics 2015,31:3210-2.

48. Andrews S. FastQC. Github, n.d.

49. Bolger AM, Lohse M, Usadel B. Trimmomatic: a flexible trimmer for Illumina sequence data. Bioinformatics 2014,30:2114-20.

50. Kolmogorov M, Yuan J, Lin Y, Pevzner PA. Assembly of long, error-prone reads using repeat graphs. Nat Biotechnol 2019,37:540-6. 
51. Lin Y, Yuan J, Kolmogorov M, Shen MW, Chaisson M, Pevzner PA. Assembly of long error-prone reads using de Bruijn graphs. Proc Natl Acad Sci U S A 2016,113:E8396-405.

52. Kajitani R, Yoshimura D, Okuno M, Minakuchi Y, Kagoshima H, Fujiyama A, et al. Platanus-allee is a de novo haplotype assembler enabling a comprehensive access to divergent heterozygous regions. Nat Commun 2019,10:1702.

53. Chakraborty M, Baldwin-Brown JG, Long AD, Emerson JJ. Contiguous and accurate de novo assembly of metazoan genomes with modest long read coverage. Nucleic Acids Res 2016,44:e147.

54. Roach MJ, Schmidt SA, Borneman AR. Purge Haplotigs: allelic contig reassignment for third-gen diploid genome assemblies. BMC Bioinformatics 2018,19:460.

55. Durand NC, Shamim MS, Machol I, Rao SSP, Huntley MH, Lander ES, et al. Juicer Provides a One-Click System for Analyzing Loop-Resolution Hi-C Experiments. Cell Syst 2016,3:95-8.

56. Dudchenko O, Batra SS, Omer AD, Nyquist SK, Hoeger M, Durand NC, et al. De novo assembly of the Aedes aegypti genome using Hi-C yields chromosome-length scaffolds. Science 2017,356:92-5.

57. Durand NC, Robinson JT, Shamim MS, Machol I, Mesirov JP, Lander ES, et al. Juicebox Provides a Visualization System for Hi-C Contact Maps with Unlimited Zoom. Cell Syst 2016,3:99-101.

58. Walker BJ, Abeel T, Shea T, Priest M, Abouelliel A, Sakthikumar S, et al. Pilon: An Integrated Tool for Comprehensive Microbial Variant Detection and Genome Assembly Improvement. PLoS One 2014,9:e112963.

59. Holt C, Yandell M. MAKER2: an annotation pipeline and genome-database management tool for second-generation genome projects. BMC Bioinformatics 2011,12:1-14.

60. Stanke M, Steinkamp R, Waack S, Morgenstern B. AUGUSTUS: a web server for gene finding in eukaryotes. Nucleic Acids Res 2004,32:W309-12.

61. Lomsadze A, Ter-Hovhannisyan V, Chernoff YO, Borodovsky M. Gene identification in novel eukaryotic genomes by self-training algorithm. Nucleic Acids Res 2005,33:6494-506.

62. Solovyev V. Statistical approaches in eukaryotic gene prediction. In: D.J. Balding, M. Bishop, C. Cannings, editor. Handbook of Statistical Genetics, Chichester: John Wiley \& Sons Ltd, 2001, p. 83-127.

63. Haas BJ, Salzberg SL, Zhu W, Pertea M, Allen JE, Orvis J, et al. Automated eukaryotic gene structure annotation using EVidenceModeler and the Program to Assemble Spliced Alignments. Genome Biol 2008,9:1-22.

64. Smit AFA, Hubley R. RepeatModeler Open-1.0 2008-2015. http://www.repeatmasker.org.

65. Wheeler TJ, Eddy SR. nhmmer: DNA homology search with profile HMMs. Bioinformatics 2013,29:2487-9.

66. Finn RD, Bateman A, Clements J, Coggill P, Eberhardt RY, Eddy SR, et al. Pfam: the protein families database. Nucleic Acids Res 2014,42:D222-30.

67. Smit AFA, Hubley R, Green P. RepeatMasker Open-4.0 2013-2015. http://www.repeatmasker.org.

68. Kim D, Langmead B, Salzberg SL. HISAT: a fast spliced aligner with low memory requirements. Nat Methods 2015,12:357-60.

69. Pertea M, Pertea GM, Antonescu CM, Chang T-C, Mendell JT, Salzberg SL. StringTie enables improved reconstruction of a transcriptome from RNA-seq reads. Nat Biotechnol 2015,33:290-5.

70. Grabherr MG, Haas BJ, Yassour M, Levin JZ, Thompson DA, Amit I, et al. Full-length transcriptome assembly from RNA-Seq data without a reference genome. Nat Biotechnol 2011,29:644-52.

71. Gilbert D. EvidentialGene - Evidence Directed Gene Construction for Eukaryotes 2013. https://sourceforge.net/projects/evidentialgene/.

72. Götz S, García-Gómez JM, Terol J, Williams TD, Nagaraj SH, Nueda MJ, et al. High-throughput functional annotation and data mining with the Blast2GO suite. Nucleic Acids Res 2008,36:3420-35.

73. Nawrocki EP, Eddy SR. Infernal 1.1: 100-fold faster RNA homology searches. Bioinformatics 2013,29:2933-5.

Page 22/37 
74. Bernt M, Donath A, Jühling F, Externbrink F, Florentz C, Fritzsch G, et al. MITOS: Improved de novo metazoan mitochondrial genome annotation. Mol Phylogenet Evol 2013,69:313-9.

75. Emms DM, Kelly S. OrthoFinder: phylogenetic orthology inference for comparative genomics. Genome Biol 2019,20:1-14.

76. Emms DM, Kelly S. STAG: Species Tree Inference from All Genes. bioRxiv 2018:267914. https://doi.org/10.1101/267914.

77. Katoh K, Standley DM. MAFFT multiple sequence alignment software version 7: improvements in performance and usability. Mol Biol Evol 2013,30:772-80.

78. Katoh K, Misawa K, Kuma K-I, Miyata T. MAFFT: a novel method for rapid multiple sequence alignment based on fast Fourier transform. Nucleic Acids Res 2002,30:3059-66.

79. Stamatakis A. RAxML version 8: a tool for phylogenetic analysis and post-analysis of large phylogenies. Bioinformatics 2014,30:1312-3.

80. Le SQ, Gascuel O. An improved general amino acid replacement matrix. Mol Biol Evol 2008,25:1307-20.

81. Robinson JT, Thorvaldsdóttir H, Winckler W, Guttman M, Lander ES, Getz G, et al. Integrative genomics viewer. Nat Biotechnol 2011,29:24-6.

82. Nelson DR. The Cytochrome P450 Homepage. Hum Genomics 2009,4:1-7.

83. UGT Committee. UGT Committee Home. UGT Committee Home n.d. https://prime.vetmed.wsu.edu/resources/udpglucuronsyltransferase-homepage (accessed March 25, 2021).

84. Pflug JM, Holmes VR, Burrus C, Spencer Johnston J, Maddison DR. Measuring genome sizes using read-depth, kmers, and flow cytometry: methodological comparisons in beetles (Coleoptera). bioRxiv 2019:761304. https://doi.org/10.1101/761304.

85. Hawkes W, Wotton K, University of Oxford and Wytham Woods Genome Acquisition Lab, Darwin Tree of Life Barcoding collective, Wellcome Sanger Institute Tree of Life programme, Wellcome Sanger Institute Scientific Operations: DNA Pipelines collective, et al. The genome sequence of the tapered dronefly, Eristalis pertinax (Scopoli, 1763). Wellcome Open Res 2021,6:292.

86. Hanrahan SJ, Johnston JS. New genome size estimates of 134 species of arthropods. Chromosome Res 2011,19:809-23.

87. Petersen M, Armisén D, Gibbs RA, Hering L, Khila A, Mayer G, et al. Diversity and evolution of the transposable element repertoire in arthropods with particular reference to insects. BMC Evol Biol 2019,19:11.

88. Chen M, Peng K, Su C, Wang Y, Hao J. The complete mitochondrial genome of Syrphus ribesii (Diptera: Syrphoidea: Syrphidae). Mitochondrial DNA B Resour 2021,6:519-21.

89. Wiegmann BM, Trautwein MD, Winkler IS, Barr NB, Kim J-W, Lambkin C, et al. Episodic radiations in the fly tree of life. Proc Natl Acad Sci U S A 2011,108:5690-5.

90. Zhao J, Xu L, Sun Y, Song P, Han Z. UDP-Glycosyltransferase Genes in the Striped Rice Stem Borer, (Walker), and Their Contribution to Chlorantraniliprole Resistance. Int J Mol Sci 2019,20. https://doi.org/10.3390/ijms20051064.

91. Tian F, Wang Z, Li C, Liu J, Zeng X. UDP-Glycosyltransferases are involved in imidacloprid resistance in the Asian citrus psyllid, Diaphorina citri (Hemiptera: Lividae). Pestic Biochem Physiol 2019,154:23-31.

92. Cui X, Wang C, Wang X, Li G, Liu Z, Wang H, et al. Molecular Mechanism of the UDP-Glucuronosyltransferase 2B20like Gene (AccUGT2B20-like) in Pesticide Resistance of Apis cerana cerana. Front Genet 2020,11:592595.

93. Ahn S-J, Marygold SJ. The UDP-Glycosyltransferase Family in Drosophila melanogaster: Nomenclature Update, Gene Expression and Phylogenetic Analysis. Front Physiol 2021,12:648481.

94. Zhou Y, Fu W-B, Si F-L, Yan Z-T, Zhang Y-J, He Q-Y, et al. UDP-glycosyltransferase genes and their association and mutations associated with pyrethroid resistance in Anopheles sinensis (Diptera: Culicidae). Malar J 2019,18:62. 
95. Xu J, Strange JP, Welker DL, James RR. Detoxification and stress response genes expressed in a western North American bumble bee, Bombus huntii (Hymenoptera: Apidae). BMC Genomics 2013,14:874.

96. Chen W, Hasegawa DK, Kaur N, Kliot A, Pinheiro PV, Luan J, et al. The draft genome of whitefly Bemisia tabaci MEAM1, a global crop pest, provides novel insights into virus transmission, host adaptation, and insecticide resistance. BMC Biol 2016,14:1-15.

97. Pan Y, Xu P, Zeng X, Liu X, Shang Q. Characterization of UDP-Glucuronosyltransferases and the Potential Contribution to Nicotine Tolerance in Myzus persicae. Int J Mol Sci 2019,20:3637.

98. Xie W, He C, Fei Z, Zhang Y. Chromosome-level genome assembly of the greenhouse whitefly (Trialeurodes vaporariorum Westwood). Mol Ecol Resour 2020,20:995-1006.

99. Guo S-K, Cao L-J, Song W, Shi P, Gao Y-F, Gong Y-J, et al. Chromosome-level assembly of the melon thrips genome yields insights into evolution of a sap-sucking lifestyle and pesticide resistance. Mol Ecol Resour 2020,20:1110-25.

100. Guo L, Xie W, Yang Z, Xu J, Zhang Y. Genome-Wide Identification and Expression Analysis of UdpGlucuronosyltransferases in the Whitefly Bemisia Tabaci (Gennadius) (HemipterA: Aleyrodidae). Int J Mol Sci 2020,21. https://doi.org/10.3390/ijms21228492.

101. Dermauw W, Pym A, Bass C, Van Leeuwen T, Feyereisen R. Does host plant adaptation lead to pesticide resistance in generalist herbivores? Curr Opin Insect Sci 2018,26:25-33.

102. Pan Y, Tian F, Wei X, Wu Y, Gao X, Xi J, et al. Thiamethoxam Resistance in Aphis gossypii Glover Relies on Multiple UDP-Glucuronosyltransferases. Front Physiol 2018,9:322.

103. Lewis JA, Szilagyi M, Gehman E, Dennis WE, Jackson DA. Distinct patterns of gene and protein expression elicited by organophosphorus pesticides in Caenorhabditis elegans. BMC Genomics 2009,10:202.

104. Lumjuan N, Rajatileka S, Changsom D, Wicheer J, Leelapat P, Prapanthadara L-A, et al. The role of the Aedes aegypti Epsilon glutathione transferases in conferring resistance to DDT and pyrethroid insecticides. Insect Biochem Mol Biol 2011,41:203-9.

105. Vontas JG, Small GJ, Hemingway J. Glutathione S-transferases as antioxidant defence agents confer pyrethroid resistance in Nilaparvata lugens. Biochem J 2001,357:65-72.

106. Corona M, Robinson GE. Genes of the antioxidant system of the honey bee: annotation and phylogeny. Insect Mol Biol 2006,15:687-701.

107. Low WY, Ng HL, Morton CJ, Parker MW, Batterham P, Robin C. Molecular evolution of glutathione S-transferases in the genus Drosophila. Genetics 2007,177:1363-75.

108. Lumjuan N, Stevenson BJ, Prapanthadara L-A, Somboon P, Brophy PM, Loftus BJ, et al. The Aedes aegypti glutathione transferase family. Insect Biochem Mol Biol 2007,37:1026-35.

109. Ding Y, Ortelli F, Rossiter LC, Hemingway J, Ranson H. The Anopheles gambiae glutathione transferase supergene family: annotation, phylogeny and expression profiles. BMC Genomics 2003,4:35.

110. Yan L, Yang P, Jiang F, Cui N, Ma E, Qiao C, et al. Transcriptomic and phylogenetic analysis of Culex pipiens quinquefasciatus for three detoxification gene families. BMC Genomics 2012,13:609.

111. Claudianos C, Ranson H, Johnson RM, Biswas S, Schuler MA, Berenbaum MR, et al. A deficit of detoxification enzymes: pesticide sensitivity and environmental response in the honeybee. Insect Mol Biol 2006,15:615-36.

112. Aidlin Harari O, Santos-Garcia D, Musseri M, Moshitzky P, Patel M, Visendi P, et al. Molecular Evolution of the Glutathione S-Transferase Family in the Bemisia tabaci Species Complex. Genome Biol Evol 2020,12:3857-72.

113. Friedman R. Genomic organization of the glutathione S-transferase family in insects. Mol Phylogenet Evol 2011,61:924-32.

114. Gawande ND, Subashini S, Murugan M, Subbarayalu M. Molecular screening of insecticides with sigma glutathione S-transferases (GST) in cotton aphid Aphis gossypii using docking. Bioinformation 2014,10:679-83.

Page 24/37 
115. Sogorb MA, Vilanova E. Enzymes involved in the detoxification of organophosphorus, carbamate and pyrethroid insecticides through hydrolysis. Toxicol Lett 2002,128:215-28.

116. Hemingway J, Ranson H. Insecticide resistance in insect vectors of human disease. Annu Rev Entomol 2000,45:37191.

117. Bisset JA, Marín R, Rodríguez MM, Severson DW, Ricardo Y, French L, et al. Insecticide resistance in two Aedes aegypti (Diptera: Culicidae) strains from Costa Rica. J Med Entomol 2013,50:352-61.

118. Oakeshott J, Claudianos C, Campbell PM. Biochemical genetics and genomics of insect esterases. Molecular Insect ... 2010.

119. Rotenberg D, Baumann AA, Ben-Mahmoud S, Christiaens O, Dermauw W, loannidis P, et al. Genome-enabled insights into the biology of thrips as crop pests. BMC Biol 2020,18:142.

120. Ramsey JS, Rider DS, Walsh TK, De Vos M, Gordon KHJ, Ponnala L, et al. Comparative analysis of detoxification enzymes in Acyrthosiphon pisum and Myzus persicae. Insect Mol Biol 2010,19 Suppl 2:155-64.

121. Xia J, Xu H, Yang Z, Pan H, Yang X, Guo Z, et al. Genome-Wide Analysis of Carboxylesterases (COEs) in the Whitefly, (Gennadius). Int J Mol Sci 2019,20. https://doi.org/10.3390/ijms20204973.

122. Karatolos N. Molecular mechanisms of insecticide resistance in the greenhouse whitefly, Trialeurodes vaporariorum. PhD. University of Exeter, 2011.

https://doi.org/https://ore.exeter.ac.uk/repository/bitstream/handle/10036/3350/KaratolosN.pdf.

123. Bass C, Field LM. Gene amplification and insecticide resistance. Pest Manag Sci 2011,67:886-90.

124. Ono M, Swanson JJ, M. Field L, Devonshire AL, D. Siegfried B. Amplification and methylation of an esterase gene associated with insecticide-resistance in greenbugs, Schizaphis graminum (Rondani) (Homoptera: Aphididae). Insect Biochem Mol Biol 1999,29:1065-73.

125. Vontas JG, Small GJ, Hemingway J. Comparison of esterase gene amplification, gene expression and esterase activity in insecticide susceptible and resistant strains of the brown planthopper, Nilaparvata lugens (Stål). Insect Mol Biol 2000,9:655-60.

126. Raymond M, Chevillon C, Guillemaud T, Lenormand T, Pasteur N. An overview of the evolution of overproduced esterases in the mosquito Culex pipiens. Philos Trans R Soc Lond B Biol Sci 1998,353:1707-11.

127. Vaughan A, Rodriguez M, Hemingway J. The independent gene amplification of electrophoretically indistinguishable B esterases from the insecticide-resistant mosquito Culex quinquefasciatus. Biochem J 1995,305 (Pt 2):651-8.

128. Merzendorfer H. Chapter One - ABC Transporters and Their Role in Protecting Insects from Pesticides and Their Metabolites. In: Cohen E, editor. Advances in Insect Physiology, vol. 46, Academic Press, 2014, p. 1-72.

129. Dermauw $W$, Van Leeuwen $T$. The $A B C$ gene family in arthropods: comparative genomics and role in insecticide transport and resistance. Insect Biochem Mol Biol 2014,45:89-110.

130. Xiao L-F, Zhang W, Jing T-X, Zhang M-Y, Miao Z-Q, Wei D-D, et al. Genome-wide identification, phylogenetic analysis, and expression profiles of ATP-binding cassette transporter genes in the oriental fruit fly, Bactrocera dorsalis (Hendel) (Diptera: Tephritidae). Comp Biochem Physiol Part D Genomics Proteomics 2018,25:1-8.

131. Lu H, Xu Y, Cui F. Phylogenetic analysis of the ATP-binding cassette transporter family in three mosquito species. Pestic Biochem Physiol 2016,132:118-24.

132. Liu S, Zhou S, Tian L, Guo E, Luan Y, Zhang J, et al. Genome-wide identification and characterization of ATP-binding cassette transporters in the silkworm, Bombyx mori. BMC Genomics 2011,12:491.

133. Figueira-Mansur J, Schrago CG, Salles TS, Alvarenga ESL, Vasconcellos BM, Melo ACA, et al. Phylogenetic analysis of the ATP-binding cassette proteins suggests a new $A B C$ protein subfamily $\mathrm{J}$ in Aedes aegypti (Diptera: Culicidae). BMC Genomics 2020,21:463. 
134. He Q, Yan Z, Si F, Zhou Y, Fu W, Chen B. ATP-Binding Cassette (ABC) Transporter Genes Involved in Pyrethroid Resistance in the Malaria Vector Anopheles sinensis: Genome-Wide Identification, Characteristics, Phylogenetics, and Expression Profile. Int J Mol Sci 2019,20. https://doi.org/10.3390/ijms20061409.

135. Pan Y, Zeng X, Wen S, Gao X, Liu X, Tian F, et al. Multiple ATP-binding cassette transporters genes are involved in thiamethoxam resistance in Aphis gossypii glover. Pestic Biochem Physiol 2020,167:104558.

136. Pym A, Singh KS, Nordgren Å, Emyr Davies TG, Zimmer CT, Elias J, et al. Host plant adaptation in the polyphagous whitefly, Trialeurodes vaporariorum, is associated with transcriptional plasticity and altered sensitivity to insecticides. BMC Genomics 2019,20:1-19.

137. Tian L, Song T, He R, Zeng Y, Xie W, Wu Q, et al. Genome-wide analysis of ATP-binding cassette (ABC) transporters in the sweetpotato whitefly, Bemisia tabaci. BMC Genomics 2017,18:1-16.

138. Feyereisen R. INSECT P450 ENZYMES. Annu Rev Entomol 1999,44:507-33.

139. Feyereisen R. Evolution of insect P450. Biochem Soc Trans 2006,34:1252-5.

140. Karunker I, Benting J, Lueke B, Ponge T, Nauen R, Roditakis E, et al. Over-expression of cytochrome P450 CYP6CM1 is associated with high resistance to imidacloprid in the B and Q biotypes of Bemisia tabaci (Hemiptera: Aleyrodidae). Insect Biochem Mol Biol 2008,38:634-44.

141. Liang X, Xiao D, He Y, Yao J, Zhu G, Zhu KY. Insecticide-mediated up-regulation of cytochrome P450 genes in the red flour beetle (Tribolium castaneum). Int J Mol Sci 2015,16:2078-98.

142. Puinean AM, Foster SP, Oliphant L, Denholm I, Field LM, Millar NS, et al. Amplification of a cytochrome P450 gene is associated with resistance to neonicotinoid insecticides in the aphid Myzus persicae. PLoS Genet 2010,6:e1000999.

143. Yang T, Liu N. Genome analysis of cytochrome P450s and their expression profiles in insecticide resistant mosquitoes, Culex quinquefasciatus. PLoS One 2011,6:e29418.

144. Scott JG. Cytochromes P450 and insecticide resistance. Insect Biochem Mol Biol 1999,29:757-77.

145. Main BJ, Everitt A, Cornel AJ, Hormozdiari F, Lanzaro GC. Genetic variation associated with increased insecticide resistance in the malaria mosquito, Anopheles coluzzii. Parasit Vectors 2018,11:225.

146. Vlogiannitis S, Mavridis K, Dermauw W, Snoeck S, Katsavou E, Morou E, et al. Reduced proinsecticide activation by cytochrome P450 confers coumaphos resistance in the major bee parasite Varroa destructor. Proc Natl Acad Sci U S A 2021,118. https://doi.org/10.1073/pnas.2020380118.

147. Scott JG, Warren WC, Beukeboom LW, Bopp D, Clark AG, Giers SD, et al. Genome of the house fly, Musca domestica L., a global vector of diseases with adaptations to a septic environment. Genome Biol 2014,15:466.

148. The genome of the model beetle and pest Tribolium castaneum. Nature 2008,452:949-55.

149. Dermauw W, Van Leeuwen T, Feyereisen R. Diversity and evolution of the P450 family in arthropods. Insect Biochem Mol Biol 2020,127:103490.

150. Ilias A, Lagnel J, Kapantaidaki DE, Roditakis E, Tsigenopoulos CS, Vontas J, et al. Transcription analysis of neonicotinoid resistance in Mediterranean (MED) populations of B. tabaci reveal novel cytochrome P450s, but no nAChR mutations associated with the phenotype. BMC Genomics 2015,16:1-23.

151. Sztal T, Chung H, Berger S, Currie PD, Batterham P, Daborn PJ. A cytochrome p450 conserved in insects is involved in cuticle formation. PLoS One 2012,7:e36544.

152. Zhang H, Zhao M, Liu Y, Zhou Z, Guo J. Identification of cytochrome P450 monooxygenase genes and their expression in response to high temperature in the alligatorweed flea beetle Agasicles hygrophila (Coleoptera: Chrysomelidae). Sci Rep 2018,8:17847.

153. Scharf ME, Parimi S, Meinke LJ, Chandler LD, Siegfried BD. Expression and induction of three family 4 cytochrome P450 (CYP4)* genes identified from insecticide-resistant and susceptible western corn rootworms, Diabrotica virgifera virgifera. Insect Mol Biol 2001,10:139-46. 
154. Shi W, Sun J, Xu B, Li H. Molecular characterization and oxidative stress response of a cytochrome P450 gene (CYP4G11) from Apis cerana cerana. Z Naturforsch C 2013,68:509-21.

155. Ingham VA, Jones CM, Pignatelli P, Balabanidou V, Vontas J, Wagstaff SC, et al. Dissecting the organ specificity of insecticide resistance candidate genes in Anopheles gambiae: known and novel candidate genes. BMC Genomics 2014,15:1018.

156. Berenbaum MR, Johnson RM. Xenobiotic detoxification pathways in honey bees. Curr Opin Insect Sci 2015,10:51-8.

157. Mao W, Rupasinghe SG, Johnson RM, Zangerl AR, Schuler MA, Berenbaum MR. Quercetin-metabolizing CYP6AS enzymes of the pollinator Apis mellifera (Hymenoptera: Apidae). Comp Biochem Physiol B Biochem Mol Biol 2009,154:427-34.

158. Johnson RM, Mao W, Pollock HS, Niu G, Schuler MA, Berenbaum MR. Ecologically appropriate xenobiotics induce cytochrome P450s in Apis mellifera. PLoS One 2012,7:e31051.

159. Zhao M, Dong Y, Ran X, Guo X, Xing D, Zhang Y, et al. Sodium channel point mutations associated with pyrethroid resistance in Chinese strains of Culex pipiens quinquefasciatus (Diptera: Culicidae). Parasit Vectors 2014,7:369.

160. Weill M, Malcolm C, Chandre F, Mogensen K, Berthomieu A, Marquine M, et al. The unique mutation in ace-1 giving high insecticide resistance is easily detectable in mosquito vectors. Insect Mol Biol 2004,13:1-7.

161. Singh OP, Dykes CL, Das MK, Pradhan S, Bhatt RM, Agrawal OP, et al. Presence of two alternative kdr-like mutations, L1014F and L1014S, and a novel mutation, V1010L, in the voltage gated Na+ channel of Anopheles culicifacies from Orissa, India. Malar J 2010,9:146.

162. Tan WL, Li CX, Wang ZM, Liu MD, Dong YD, Feng XY, et al. First detection of multiple knockdown resistance (kdr)-like mutations in voltage-gated sodium channel using three new genotyping methods in Anopheles sinensis from Guangxi Province, China. J Med Entomol 2012,49:1012-20.

163. Liu N, Pridgeon JW. Metabolic detoxication and the kdr mutation in pyrethroid resistant house flies, Musca domestica (L.). Pestic Biochem Physiol 2002,73:157-63.

164. Williamson MS, Martinez-Torres D, Hick CA, Devonshire AL. Identification of mutations in the houseflypara-type sodium channel gene associated with knockdown resistance $(\mathrm{kdr})$ to pyrethroid insecticides. Mol Gen Genet 1996,252:51-60.

165. Vontas JG, Hejazi MJ, Hawkes NJ, Cosmidis N, Loukas M, Janes RW, et al. Resistance-associated point mutations of organophosphate insensitive acetylcholinesterase, in the olive fruit fly Bactrocera oleae. Insect Mol Biol 2002,11:32936.

166. Kozaki T, Shono T, Tomita T, Kono Y. Fenitroxon insensitive acetylcholinesterases of the housefly, Musca domestica associated with point mutations. Insect Biochem Mol Biol 2001,31:991-7.

167. da Silva NM, de Carvalho RA, de Azeredo-Espin AML. Acetylcholinesterase cDNA sequencing and identification of mutations associated with organophosphate resistance in Cochliomyia hominivorax (Diptera: Calliphoridae). Vet Parasitol 2011,177:190-5.

168. Ffrench-Constant RH, Rocheleau TA, Steichen JC, Chalmers AE. A point mutation in a Drosophila GABA receptor confers insecticide resistance. Nature 1993,363:449-51.

169. Scott JG. Evolution of resistance to pyrethroid insecticides in Musca domestica. Pest Manag Sci 2017,73:716-22.

170. Yang C, Huang Z, Li M, Feng X, Qiu X. RDL mutations predict multiple insecticide resistance in Anopheles sinensis in Guangxi, China. Malar J 2017,16:482.

171. Troczka B, Zimmer CT, Elias J, Schorn C, Bass C, Davies TGE, et al. Resistance to diamide insecticides in diamondback moth, Plutella xylostella (Lepidoptera: Plutellidae) is associated with a mutation in the membranespanning domain of the ryanodine receptor. Insect Biochem Mol Biol 2012,42:873-80. 
172. Lueke B, Douris V, Hopkinson JE, Maiwald F, Hertlein G, Papapostolou K-M, et al. Identification and functional characterization of a novel acetyl-CoA carboxylase mutation associated with ketoenol resistance in Bemisia tabaci. Pestic Biochem Physiol 2020,166:104583.

173. Pan Y, Zhu E, Gao X, Nauen R, Xi J, Peng T, et al. Novel mutations and expression changes of acetyl-coenzyme A carboxylase are associated with spirotetramat resistance in Aphis gossypii Glover. Insect Mol Biol 2017,26:383-91.

174. Hardstone MC, Scott JG. Is Apis mellifera more sensitive to insecticides than other insects? Pest Manag Sci 2010,66:1171-80.

175. SchmehI DR, Teal PEA, Frazier JL, Grozinger CM. Genomic analysis of the interaction between pesticide exposure and nutrition in honey bees (Apis mellifera). J Insect Physiol 2014,71:177-90.

176. Manjon C, Troczka BJ, Zaworra M, Beadle K, Randall E, Hertlein G, et al. Unravelling the Molecular Determinants of Bee Sensitivity to Neonicotinoid Insecticides. Curr Biol 2018,28:1137-43.e5.

177. Calvo-Agudo M, González-Cabrera J, Picó Y, Calatayud-Vernich P, Urbaneja A, Dicke M, et al. Neonicotinoids in excretion product of phloem-feeding insects kill beneficial insects. Proc Natl Acad Sci U S A 2019,116:16817-22.

\section{Figures}

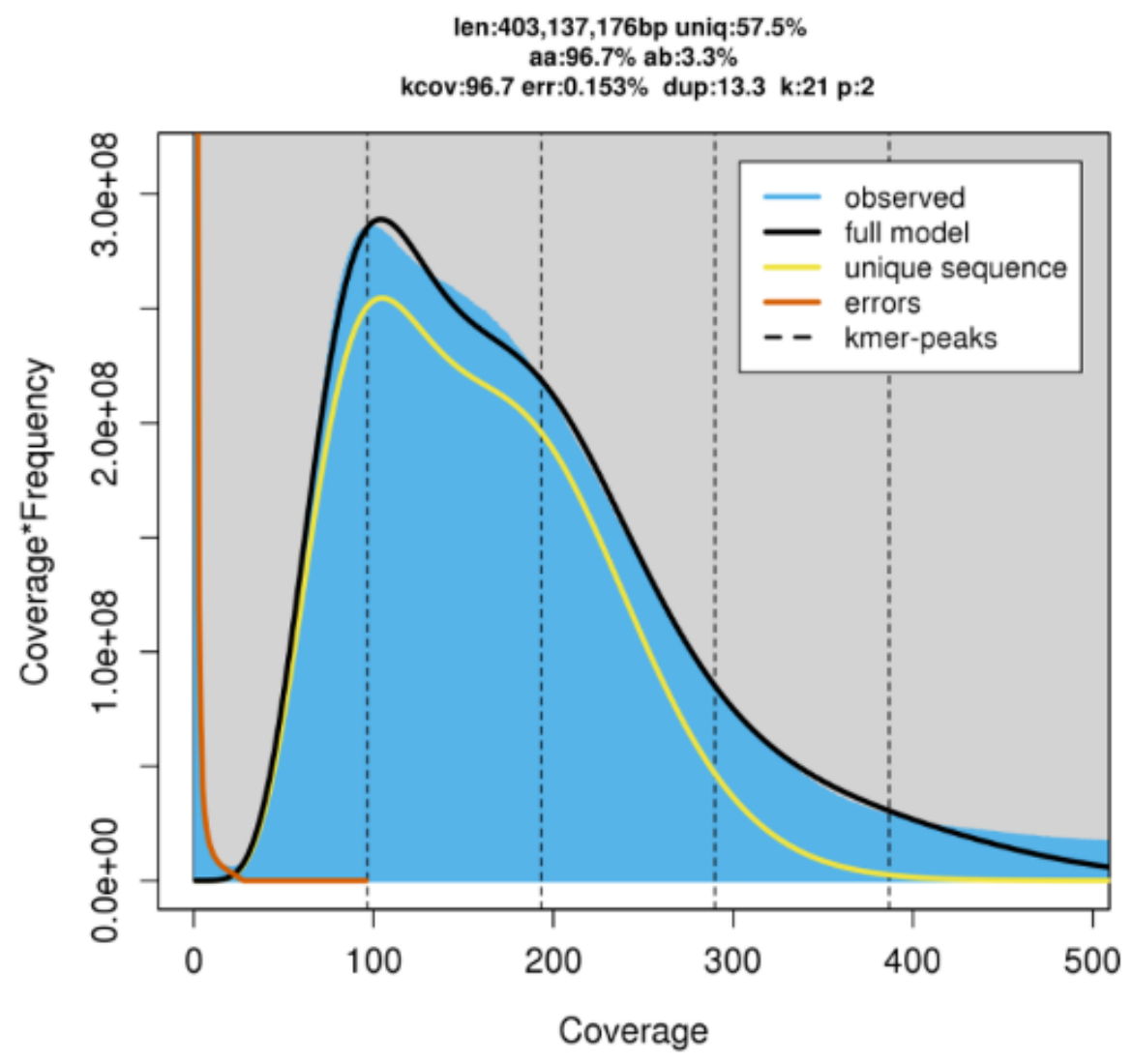

\section{Figure 1}

GenomeScope v2.0 k-mer profile plot for the S. rueppellii genome, based on 21-mers in Illumina reads. The observed k-mer frequency distribution is depicted in blue, whereas the GenomeScope fitmodel is shown as a black line. The unique and putative error k-mer distributions are plotted in yellow and red, respectively. 


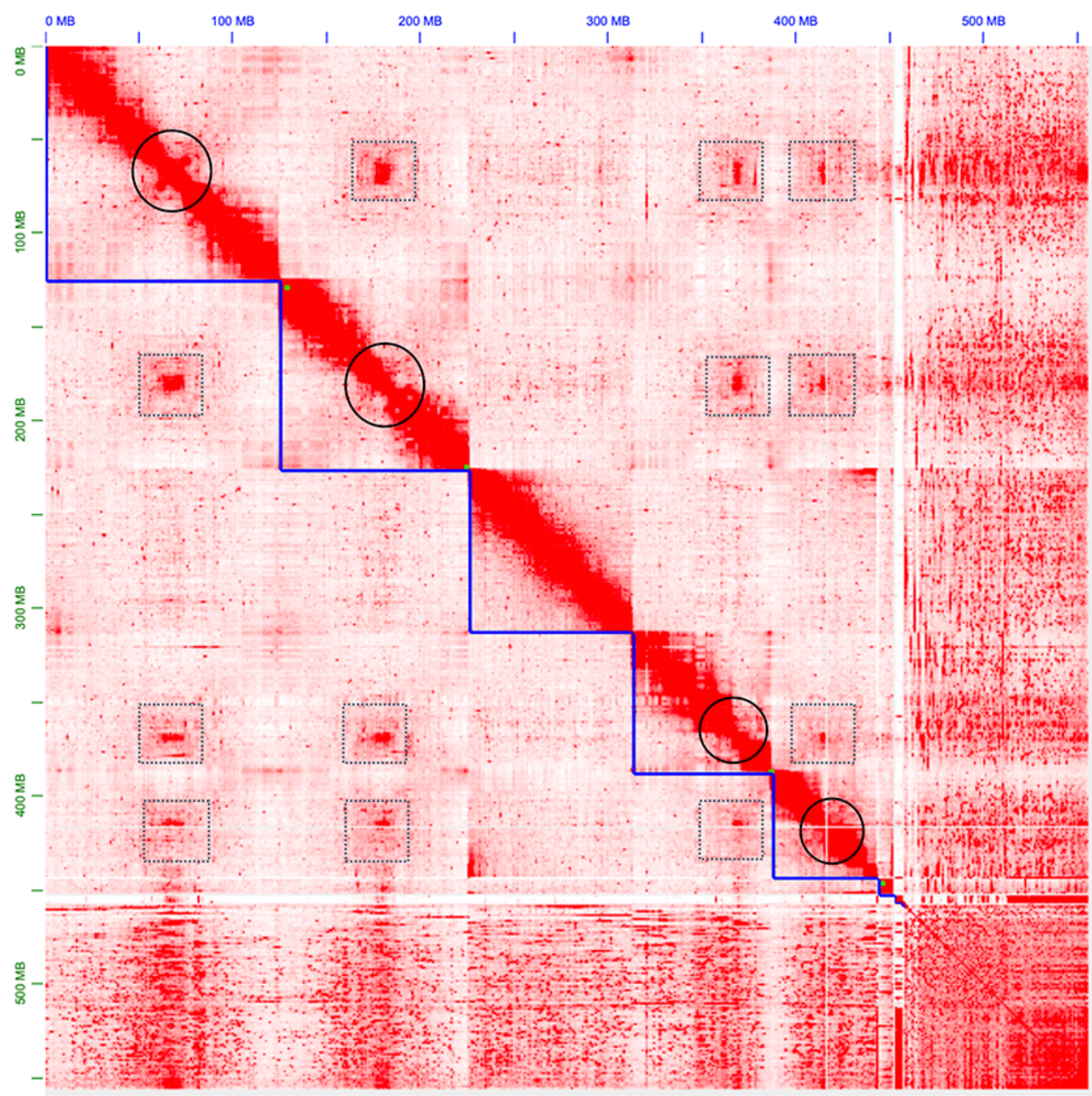

assembly

\section{Figure 2}

The Sphaerophoria rueppellii genome visualised in JuiceBox, with $\mathrm{Hi}-\mathrm{C}$ contacts shown in red. Blue edges = superscaffolds/potential chromosomes. Black circles = likely centromeres. Grey boxes = centromere - centromere interchromosomal interactions. (Potential chromosome 3 had no obvious centromere, which may have been due to a misassembly. The 7th scaffold was mostly repeat regions - evidenced by the lack of interactions with the rest of the genome.) 


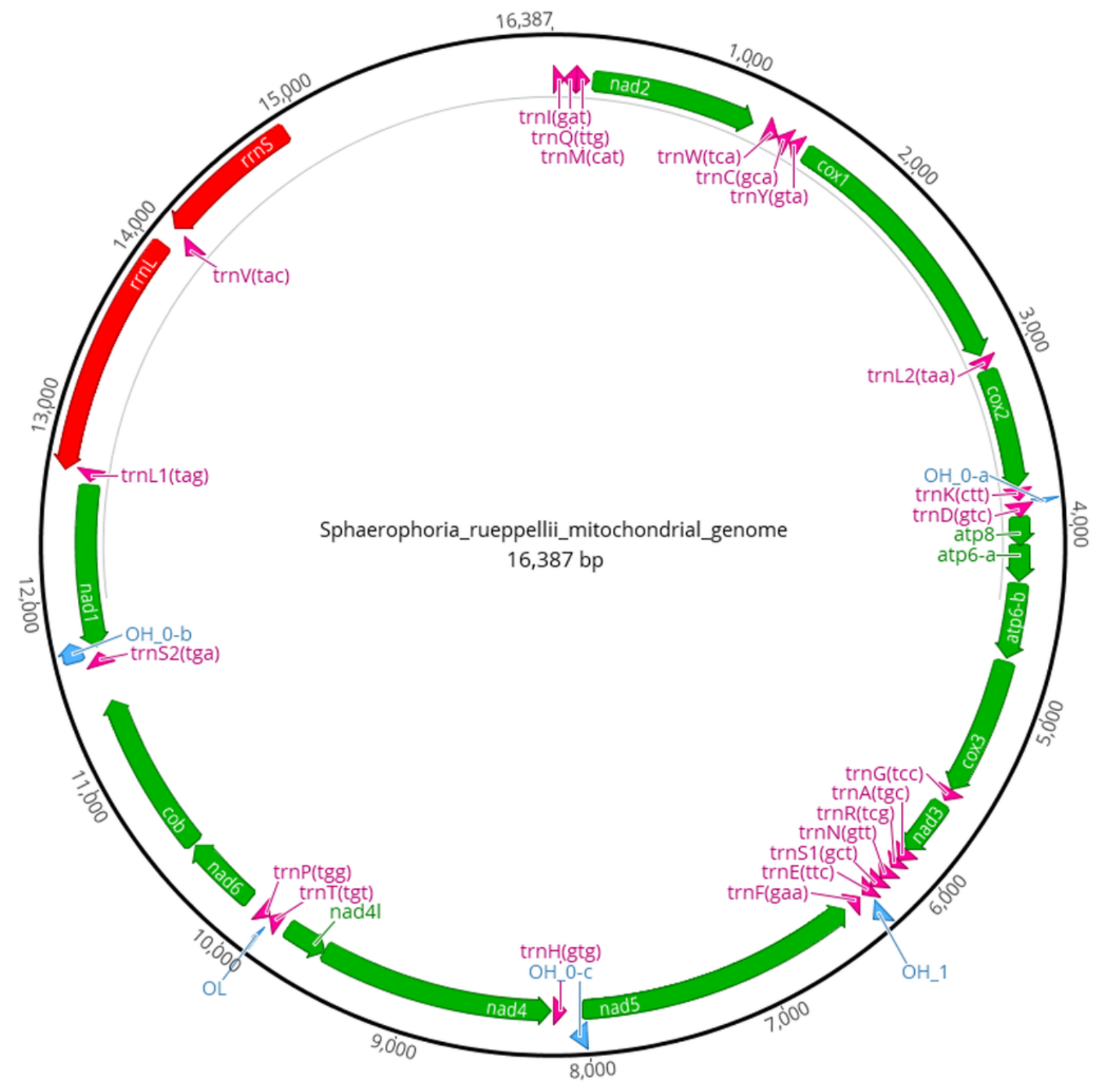

Figure 3

The mitochondrial genome for Sphaerophoria rueppellii, visualised using Geneious and annotation track obtained using MITOS2. 


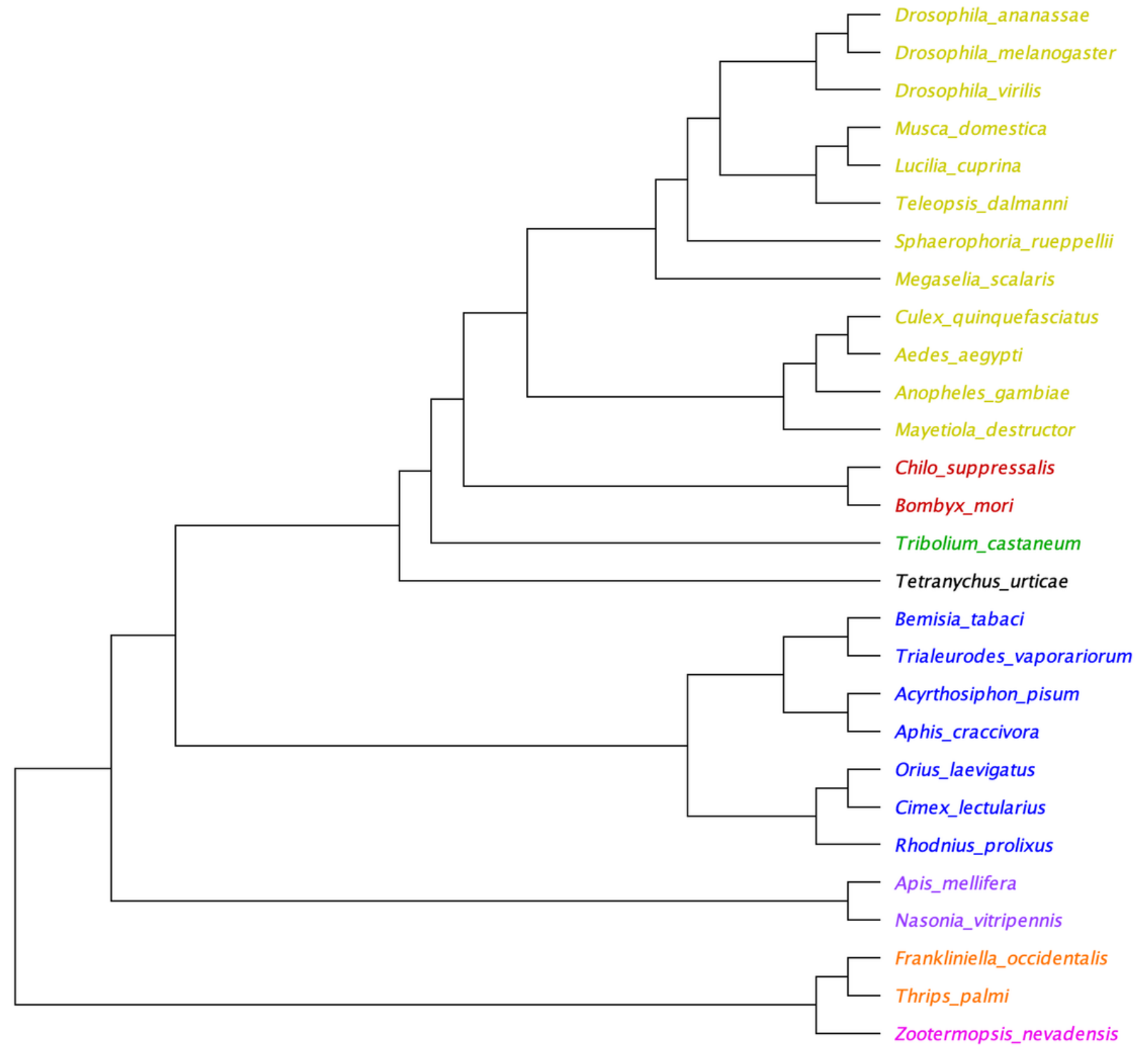

\section{Figure 4}

Phylogeny and divergence of Insecta, including crop pests and other beneficial predators. Nodes are coloured by order, yellow=Diptera, red=Lepidoptera, green=Coleoptera, black=Chelicerata, blue=Hemiptera, purple=Hymenoptera, orange=Thysanoptera, pink=Isoptera. Produced using the STAG tree inference method and full proteomes of the following species: D. ananassae: PRJNA12651, D. melanogaster: PRJNA13812, D. virilis: PRJNA12688, M. domestica:

PRJNA176013, L. cuprina: PRJNA248412, T. dalmanni: PRJNA391339, S. rueppellii: (this study), M. scalaris: PRJEB1273, C. quinquefasciatus: PRJNA18751, A. aegypti: PRJNA318737, A. gambiae: PRJNA1438, M. destructor: PRJNA45867, C. suppressalis: PRJNA506136, B. mori: PRJNA205630, T. castaneum: PRJNA12540, T. urticae: PRJNA315122, B. tabaci: PRJNA312470, T. vaporariorum: PRJNA553773, A. pisum: PRJNA13657, A. craccivora: PRJNA558689, 0. laevigatus: PRJNA721944, C. lectularius: PRJNA167477, R. prolixus: PRJNA13648, A. mellifera: PRJNA471592, N. vitripennis: PRJNA575073, F. occidentalis: PRJNA203209, T. palmi: PRJNA607431, Z. nevadensis: PRJNA203242. 


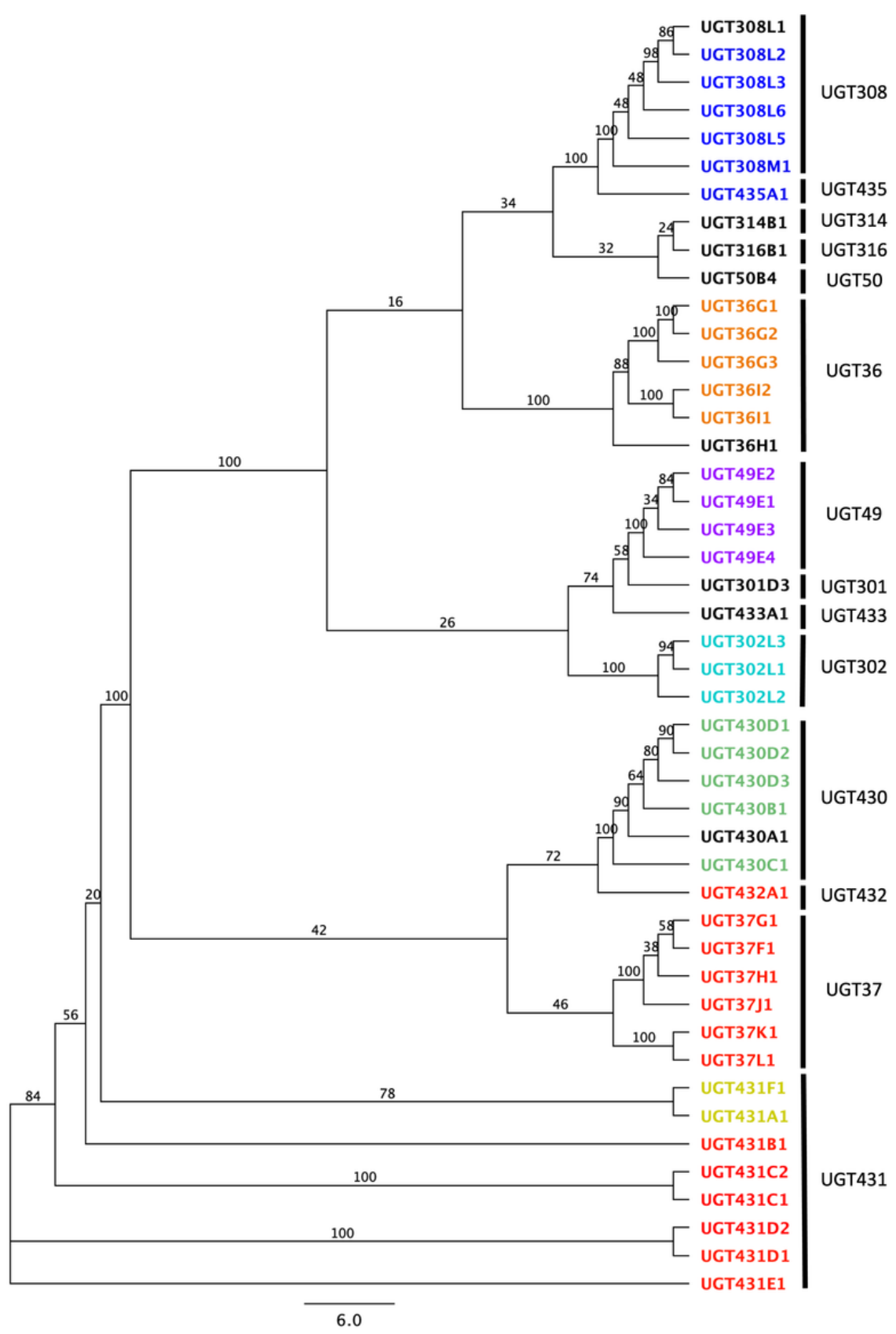

Figure 5

Phylogenetic tree of S. rueppellii UDP-glycosyltransferases. Amino acid sequences were aligned using MAFFT and analyzed using RAxML (the GAMMA LG protein model was used). The bootstrap consensus tree was inferred from 100 replicates. Coloured nodes indicate groups of likely recent tandem duplications, based on genes within the cluster having $>70 \%$ similarity using Blosum45 with threshold 0, and being located adjacently in the genome. 


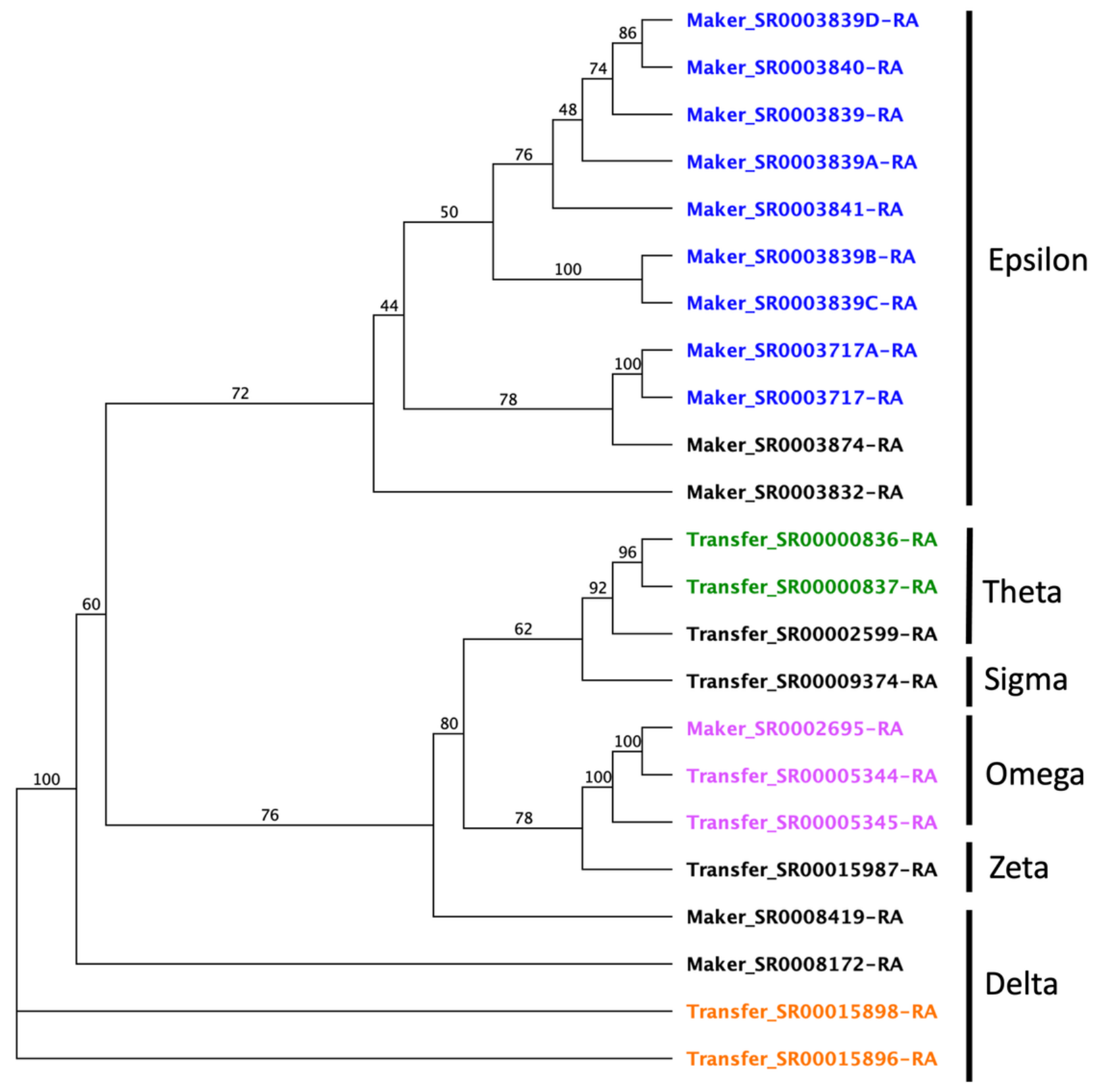

Figure 6

Phylogenetic tree of the Sphaerophoria rueppellii glutathione S-transferases. Amino acid sequences were aligned using MAFFT and analyzed using RAXML (the GAMMA LG protein model was used). The bootstrap consensus tree was inferred from 100 replicates. Coloured nodes indicate groups of likely recent tandem duplications, based on genes within the cluster having $>70 \%$ similarity using Blosum 45 with threshold 0 , and being located adjacently in the genome. 


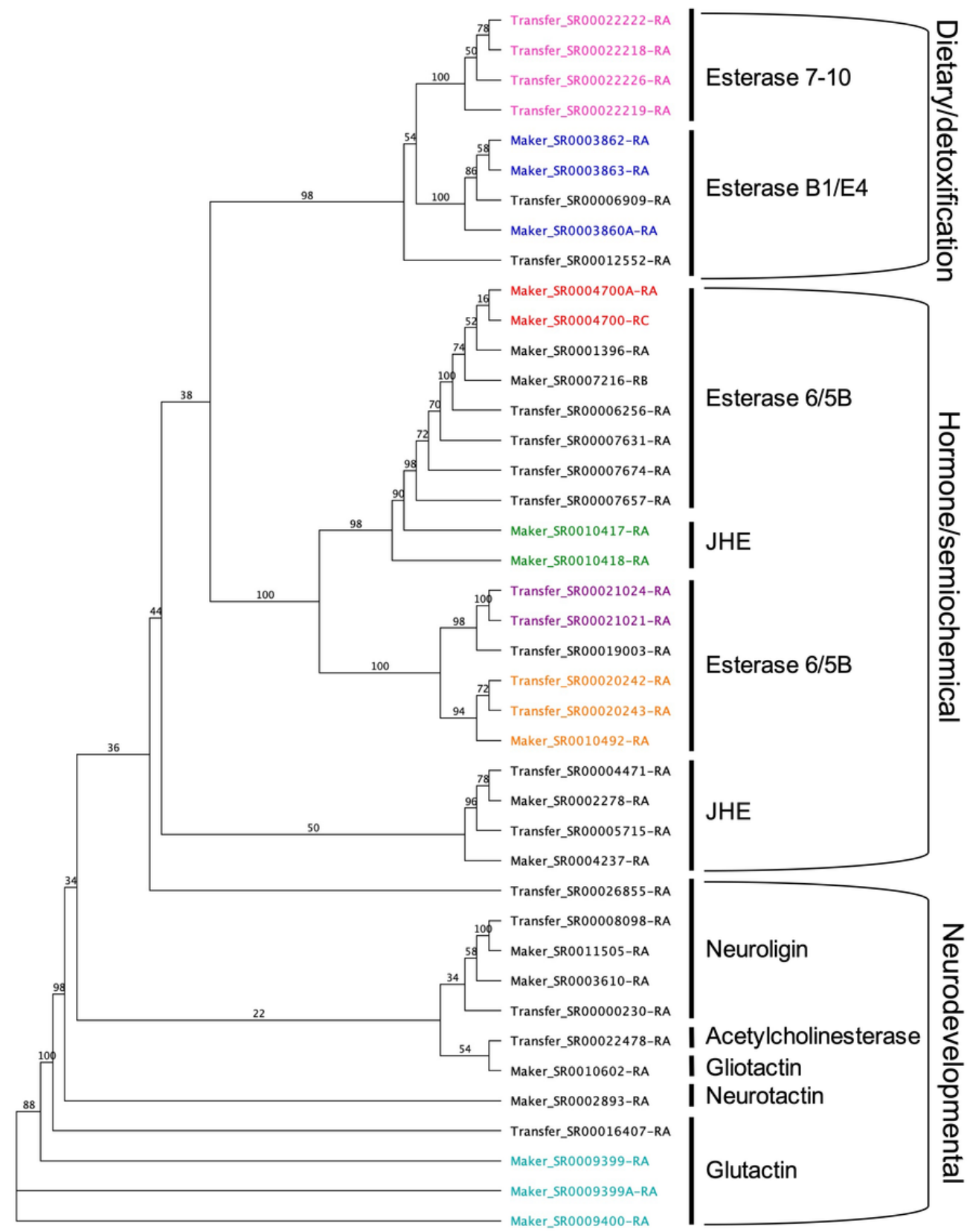

Figure 7

Phylogenetic tree of the Sphaerophoria rueppellii carboxyl/cholinesterases. Amino acid sequences were aligned using MAFFT and analyzed using RAXML (the GAMMA LG protein model was used). The bootstrap consensus tree was inferred from 100 replicates. Coloured nodes indicate groups of likely recent tandem duplications, based on genes within the cluster having $>70 \%$ similarity using Blosum 45 with threshold 0 , and being located adjacently in the genome.

Maker_SR0001396-RA was a gene fragment, and was not included in the final gene count or analysis, all others are fulllength genes. 


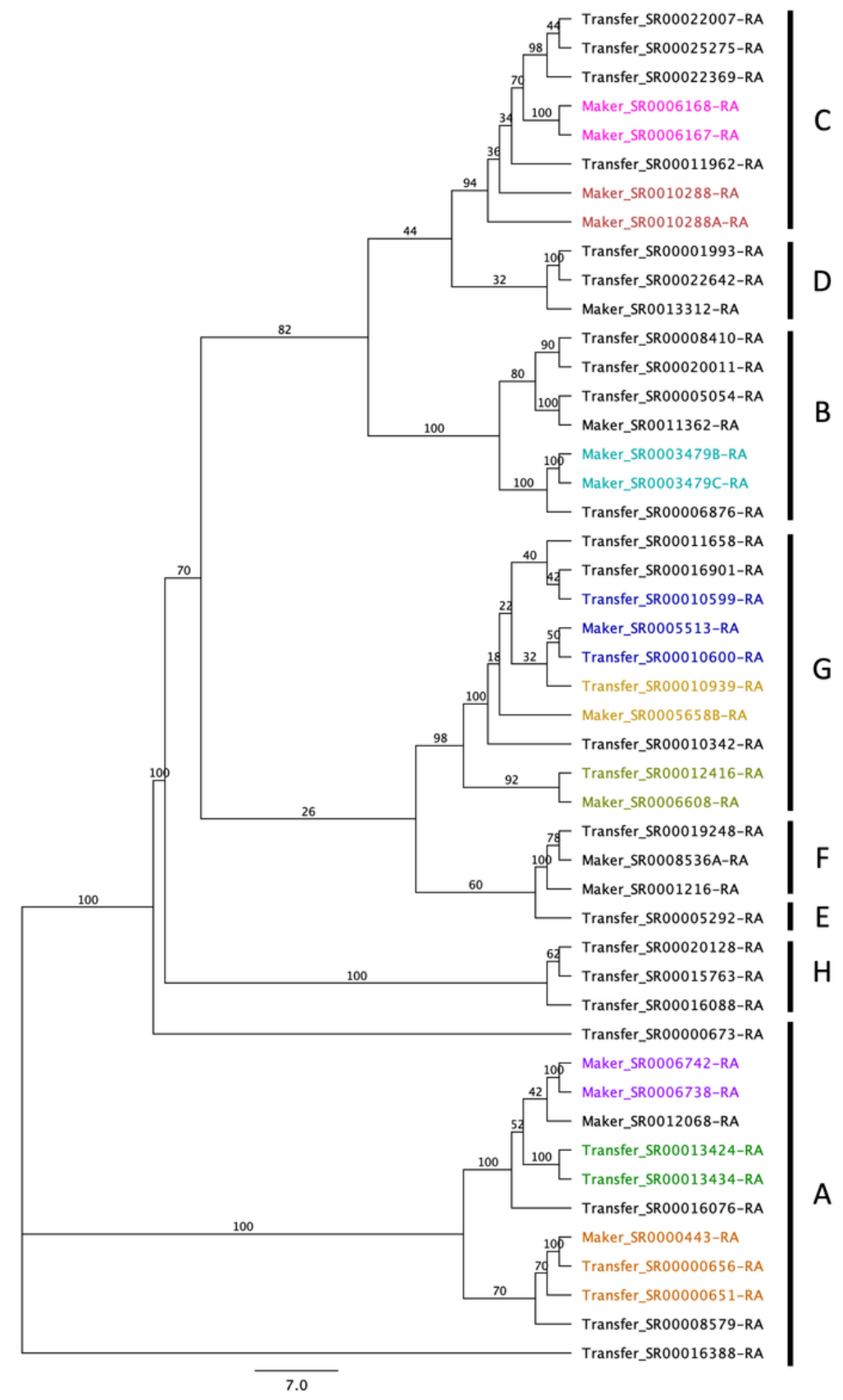

\section{Figure 8}

Phylogenetic tree of the Sphaerophoria rueppellii ABC transporters. Amino acid sequences were aligned using MAFFT and analyzed using RAXML (the GAMMA LG protein model was used). The bootstrap consensus tree was inferred from 100 replicates. Coloured nodes indicate groups of likely recent tandem duplications, based on genes within the cluster having $>70 \%$ similarity using Blosum45 with threshold 0, and being located adjacently in the genome. 


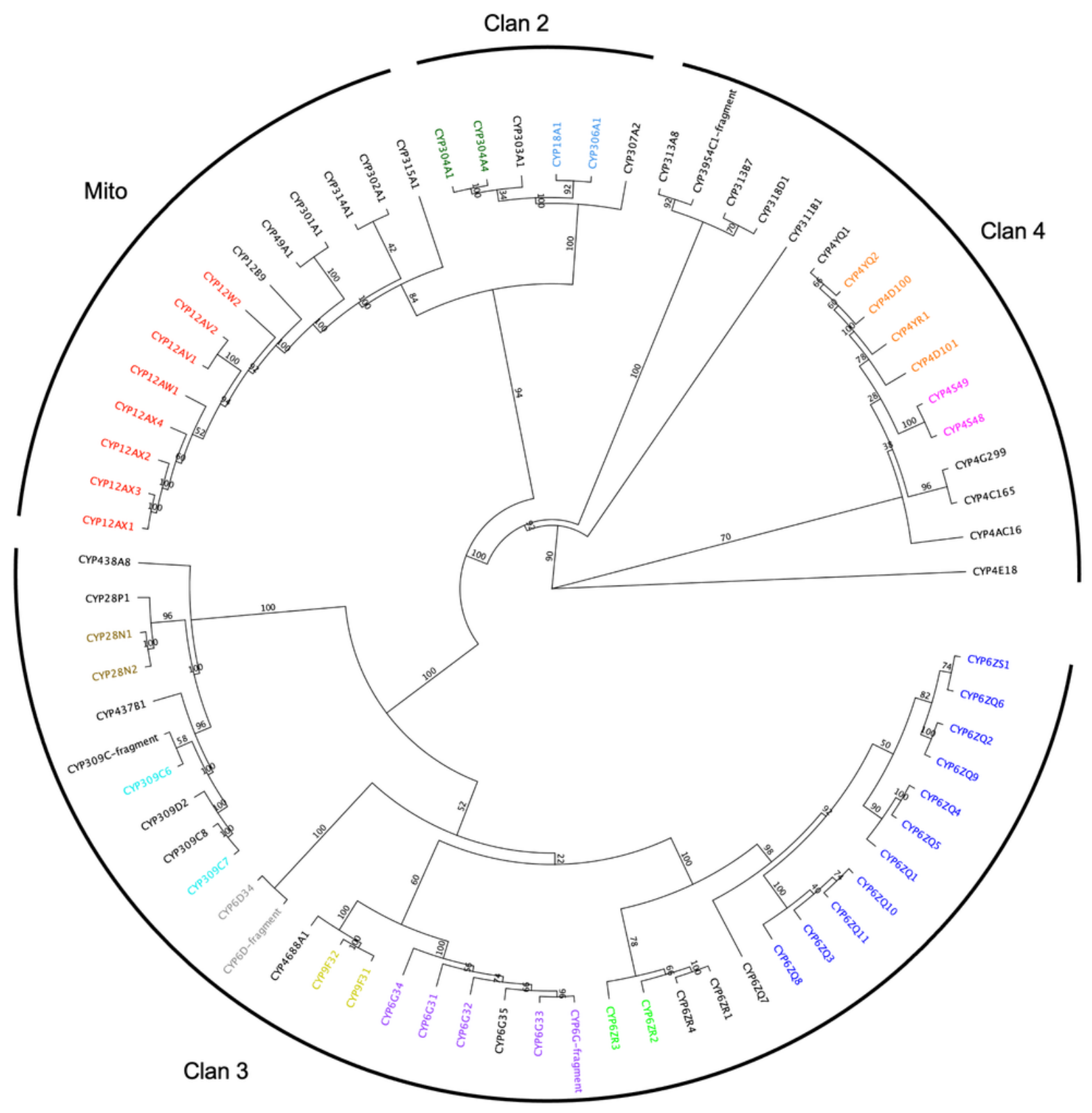

Figure 9

Phylogenetic tree of the Sphaerophoria rueppellii Cytochrome P450s. Amino acid sequences were aligned using MAFFT and analyzed using RAxML (the GAMMA LG protein model was used). The bootstrap consensus tree was inferred from 100 replicates. Coloured nodes indicate groups of likely recent tandem duplications, based on genes within the cluster having $>70 \%$ similarity using Blosum 45 with threshold 0 , and being located adjacently in the genome. The CYP6Zx family is part of clan 3 .

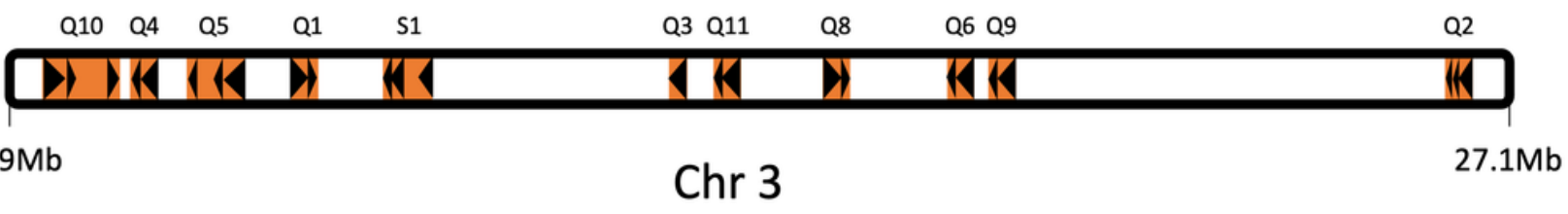




\section{Figure 10}

Arrangement of the CYP6Zx subfamily on chromosome 3. Orange boxes represent genes, black arrows represent exons as well as gene orientation.

\section{Supplementary Files}

This is a list of supplementary files associated with this preprint. Click to download.

- Additionalfile1.xlsx 